\title{
Optimization of classical hydraulic engine mounts based on RMS method
}

\author{
J. Christopherson and G. Nakhaie Jazar* \\ Department of Mechanical Engineering and Applied Mechanics, North Dakota State University, Fargo, ND 58105, \\ USA
}

Received 1 March 2004

Revised 18 June 2004

\begin{abstract}
Based on RMS averaging of the frequency response functions of the absolute acceleration and relative displacement transmissibility, optimal parameters describing the hydraulic engine mount are determined to explain the internal mount geometry. More specifically, it is shown that a line of minima exists to define a relationship between the absolute acceleration and relative displacement transmissibility of a sprung mass using a hydraulic mount as a means of suspension. This line of minima is used to determine several optimal systems developed on the basis of different clearance requirements, hence different relative displacement requirements, and compare them by means of their respective acceleration and displacement transmissibility functions. In addition, the transient response of the mount to a step input is also investigated to show the effects of the optimization upon the time domain response of the hydraulic mount.
\end{abstract}

\section{Introduction}

Hydraulic engine mounts (HEM) are seeing increased use in today's vehicles noting the trend towards lighter fourcylinder, front-wheel drive vehicles [1]. The engines utilized typically exhibit excitation frequencies in the range of $25-200 \mathrm{~Hz}$ with amplitudes generally less than $0.3 \mathrm{~mm}$ [2]. In addition, the engine mounts are subject to excitations caused by rough road surfaces. The excitation induced courtesy of rough road surfaces typically is low frequency, usually under $30 \mathrm{~Hz}$, and high-amplitude in nature [1]. Therefore, the engine mount must serve two seemingly contradictory functions, controlling engine behavior due to road excitations and isolating engine-induced vibrations by providing variable damping and stiffness across the entire frequency spectrum. For this reason, the engine mount must provide a frequency dependent and amplitude dependent behavior such that at high-amplitude/low frequency excitations the mount should provide high stiffness and damping characteristics, where as at low-amplitude/high frequency excitations the mount should provide low stiffness and damping characteristics [3].

In order to accomplish an amplitude and frequency dependent behavior, the mount must contain a variable damping mechanism that is sensitive to input excitations, in terms of both amplitude and frequency. The most common method in passive hydraulic engine mounts is the use of a device known as the decoupler, illustrated in Fig. 1. The decoupler is simply a floating disk contained within a cage limiting its travel. The reason such a device provides amplitude and frequency dependent behavior is that under high amplitude-low excitation frequency vibrations, the decoupler closes due to the pressure differential in the chambers of the hydraulic mount shown in Fig. 1. Once the decoupler closes (impacts the cage bounds), very minimal, if any, flow is allowed through forcing the pressure differential in the fluid chambers to be equalized by flow through a long, small diameter tube known as the inertia track. The inertia track, noting its dimensions with respect to the decoupler, causes a highly restrictive flow path thereby increasing the

\footnotetext{
* Corresponding author: G. Nakhaie Jazar, Department of Mechanical Engineering and Applied Mechanics, North Dakota State University, Fargo, ND 58105-5285, USA. Tel.: +1 701231 8303; Fax: +1 701231 8913; E-mail: Reza.N.Jazar@ndsu.nodak.edu.
} 


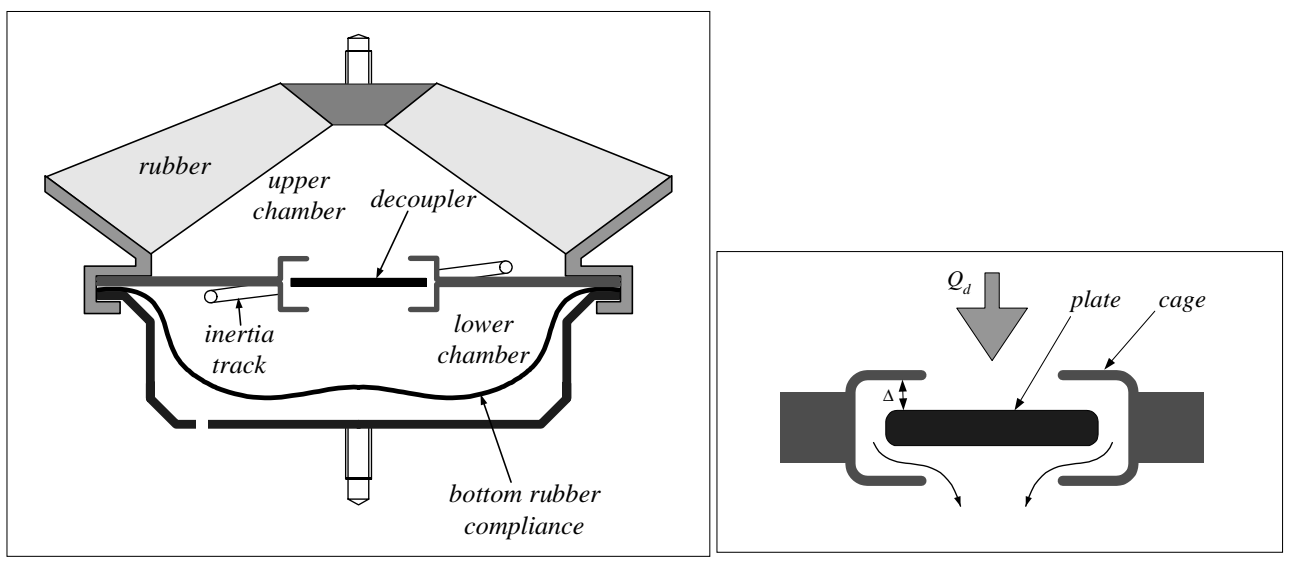

Fig. 1. Standard type hydraulic engine mount.

damping coefficient associated with the mount. However, when the mount undergoes low amplitude-high excitation frequency vibrations, the decoupler does not come into contact with the cage bounds meaning that fluid may freely pass through it in order to equalize any pressure difference between chambers thereby providing a minimal damping coefficient associated with the mount. This dual damping property provides a good means by which to alter the transmissibility of the mount by providing high damping during low frequency excitations and low damping during high frequency excitations. If one considers the behavior of a linear single degree of freedom (s.d.o.f.) system across the frequency spectrum the need for such a device clearly illustrates itself as is visible in Fig. 2 illustrating the transmitted force to the sprung mass of a base excited system [4].

As may be seen in Fig. 2, the s.d.o.f. mount undergoes very favorable behavior during low frequency excitations assuming a high viscous damping coefficient $(\xi)$. However, if the damping coefficient remains constant, the mount transmissibility grows excessively as excitation frequency increases. On the other hand, if a mount with a very low viscous damping coefficient is utilized; such a mount exhibits excessive transmissibility through the low excitation frequency spectrum. However, as the excitation frequency increases such a mount exhibits lower transmissibility than the similar mount with high damping. Therefore, the hydraulic engine mount is utilized to provide a dual mode damping that is relatively high during low frequency excitations due to the decoupler closing and relatively low during high frequency excitations.

Because the shift from high to low damping modes of the hydraulic engine mount depends strongly on the dimensions of the decoupler, it becomes quite important when undertaking any optimization process to consider the decoupler geometry. However, the decoupler geometry alone does not fully control the switching point between high and low damping, the inertia track geometry is also important. For example, if the inertia track is too large in cross-sectional area, it will fail to provide sufficient additional damping to the system when the decoupler closes. In addition, the inertia track will then provide a means for more rapid equalization of pressure between chambers noting the lower resistance to flow provided by the larger cross-sectional area. However, if the inertia track is too small, the fluid may not be able to flow sufficiently through the inertia track to equalize the pressure difference between chambers in a rapid manner, causing the mount to behave as if it just contained the decoupler. This type of inertia track geometry may not allow the damping of the mount to decrease as the excitation frequency increases; therefore, the mount behavior would suffer at high frequency excitations. This is why the internal geometry of the mount must be considered as a whole and optimized in a manner that will provide the desired switching behavior.

\section{Mathematical model}

In order to optimize the behavior of the HEM as an overall response of a base excited system, an appropriate mathematical model must be developed using the lumped parameter model shown in Fig. 3. However, to utilize such a lumped parameter model a method of determining the equivalent fluid mass and flow resistance of both the 


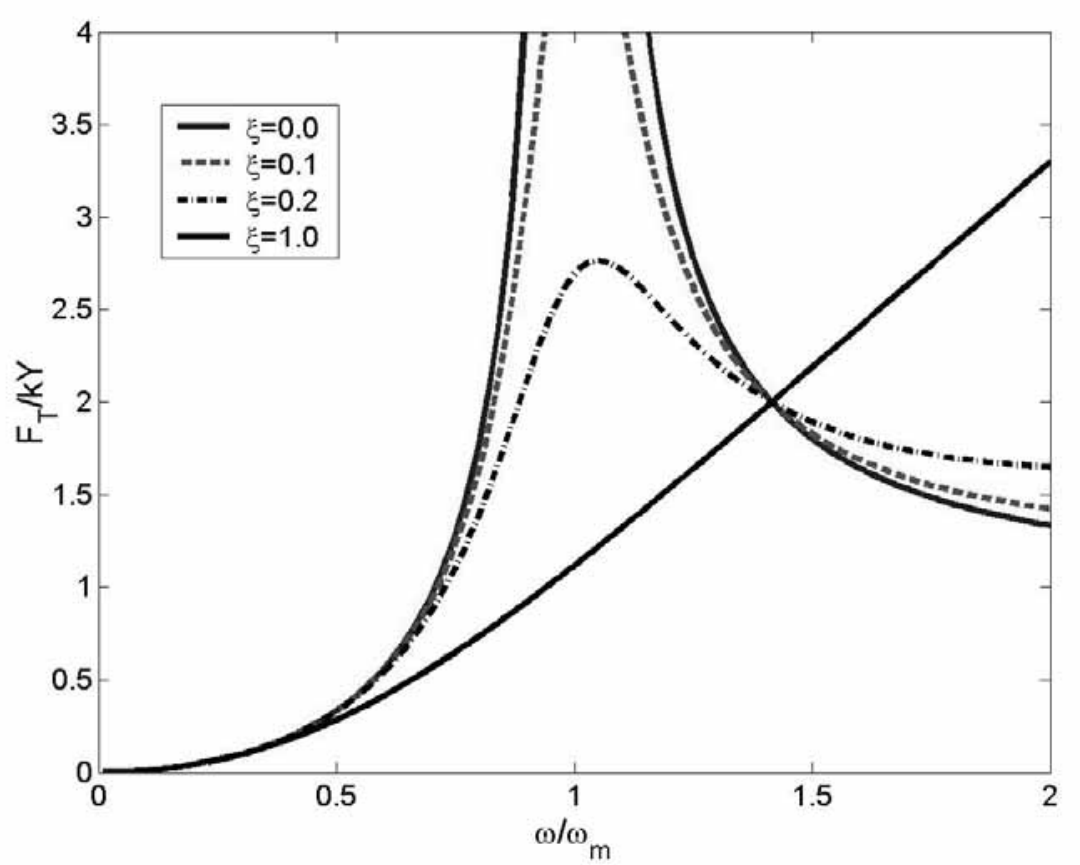

Fig. 2. Typical single degree of freedom mount transmissibility frequency response.

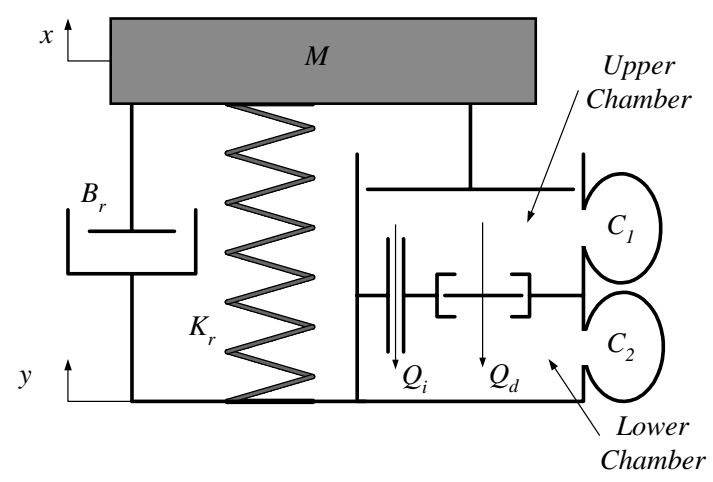

Fig. 3. Lumped parameter model of the hydraulic engine mount.

decoupler and inertia track must be developed. Literature to date has shown good results utilizing the following relationships to describe said quantities [5].

$$
\begin{aligned}
& N_{d}=\begin{array}{c}
128 \mu L_{d} \\
\pi D_{d}^{4}
\end{array}, N_{i}=\begin{array}{c}
128 \mu L_{i} \\
\pi D_{i}^{4}
\end{array} \\
& B_{d}=N_{d} A_{d}^{2}, B_{i}=N_{i} A_{i}^{2} \\
& M_{d}=\rho L_{d} A_{d}, M_{i}=\rho L_{i} A_{i}
\end{aligned}
$$

Equations (1) and (2) provide a means to describe the flow resistance through a fluid channel in the engine mount. Equation (3) provides a means for calculating an effective mass for the fluid columns. With these equations, it is now possible to determine the equations of motion utilizing the lumped parameter model. 


$$
\begin{aligned}
& Q_{d}=A_{d} \dot{x}_{d} \\
& Q_{i}=A_{i} \dot{x}_{i} \\
& M_{d} \ddot{x}_{d}+\left(B_{d}+E \begin{array}{r}
x_{d}^{n} \\
\Delta^{n}
\end{array}\right) \dot{x}_{d}=A_{d}\left(P_{1}-P_{2}\right) \\
& M_{i} \ddot{x}_{i}+B_{i} \dot{x}_{i}=A_{i}\left(P_{1}-P_{2}\right) \\
& A_{p}(\dot{x}-\dot{y})=Q_{i}+Q_{d}+C_{1}\left(\dot{P}_{1}-\dot{P}_{a t m}\right) \\
& Q_{d}+Q_{i}=C_{2}\left(\dot{P}_{2}-\dot{P}_{a t m}\right)
\end{aligned}
$$

Equations (4) and (5) describe the fluid flow rate through the decoupler and inertia track respectively. Equations (6) and (7) are utilized to describe the conservation of momentum through the fluid channels. In addition, Eq. (6) contains the nonlinear function developed by Jazar and Golnaraghi to describe the decoupler closing event by providing a variable flow resistance dependent upon the position of the decoupler [3,6]. Equations (8) and (9) ensure continuity in the system and are utilized in conjunction with the flow rate and conservation of momentum equations to arrive at the final system of equations. However, to fully describe the system, the behavior of the engine mass must be considered as follows.

$$
M \ddot{x}+B_{r}(\dot{x}-\dot{y})+K_{r}(x-y)+A_{p} P_{1}=0
$$

Equation (10) defines the behavior of the engine mass supported by the HEM while undergoing base excitation as is shown in Fig. 3.

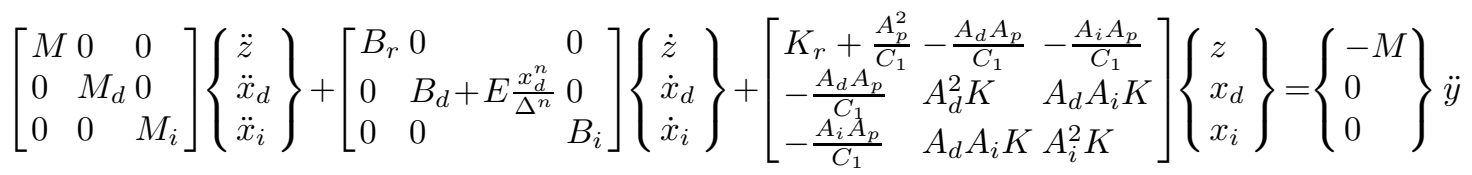

where:

$$
z=x-y, y=Y \sin (\omega \cdot t)
$$

Equations (11) and (12) describe the behavior of the engine mass supported by the HEM in conjunction with the behavior of the fluid in the internal passages of the mount. However, to make these equations of motion suitable for perturbation analysis, the equations must be written in a nondimensional form by introducing the following nondimensional parameters.

$$
\begin{gathered}
\tau=\Omega t, \Omega^{2}=\begin{array}{c}
A_{d}^{2} K \\
M_{d}
\end{array}, x_{d}=\Delta u_{d}, x_{i}=\Delta u_{i}, z=Y q, w=\begin{array}{c}
\omega \\
\Omega
\end{array} \\
\zeta=\begin{array}{c}
B_{r} \\
M \Omega
\end{array}, \zeta_{d}=\begin{array}{c}
B_{d} \\
M_{d} \Omega
\end{array}, \zeta_{i}=\begin{array}{c}
B_{i} \\
M_{i} \Omega
\end{array}, e=\begin{array}{c}
E \\
M_{d} \Omega
\end{array}, m_{d}=\begin{array}{c}
M_{d} \\
M
\end{array}, \\
m=\begin{array}{c}
M_{i} \\
M_{d}
\end{array}, a=\begin{array}{c}
A_{i} \\
A_{d}
\end{array}, a_{p}=\begin{array}{c}
A_{p} \\
A_{d}
\end{array}, f=\begin{array}{l}
Y \\
\Delta
\end{array}, g=\begin{array}{c}
K_{r} C_{1}+A_{p}^{2} \\
A_{d}^{2} K C_{1}
\end{array}, k=\begin{array}{c}
1 \\
C_{1} K
\end{array}
\end{gathered}
$$

Equation (13) introduces the time transformation parameter $\Omega$ by considering this property to be equivalent to the linear model natural frequency of the decoupler fluid column. The advantage of using this parameter opposed to any other is that by using this parameter the remainder of the nondimensional parameters containing $\Omega$ are forced to be functions of the decoupler geometry; hence, the decoupler mass and area. This advantage will be realized later during the optimization process.

Utilizing the parameters in Eq. (13), the equations of motion given in Eqs (11) and (12) may be expressed as follows. 


$$
\begin{aligned}
& q^{\prime \prime}+\zeta q^{\prime}+m_{d} g q-{ }_{f}^{m_{d} a_{p} k} u_{d}-{ }_{f}^{a a_{p} m_{d} k} u_{i}=w^{2} \sin (w \tau) \\
& u_{d}^{\prime \prime}+\left(\zeta_{d}+e u_{d}^{n}\right) u_{d}^{\prime}-a_{p} f k q+u_{d}+a u_{i}=0 \\
& u_{i}^{\prime \prime}+\zeta_{i} u_{i}^{\prime}-{ }_{m}^{a a_{p} f k} q+{ }_{m}^{a} u_{d}+{ }_{m}^{a^{2}} u_{i}=0
\end{aligned}
$$

To make Eqs (14) through (16) suitable for perturbation analysis the following nondimensional parameters are introduced.

$$
\begin{aligned}
& \varepsilon=a, \varepsilon \omega_{m}=m_{d} g, h=\begin{array}{c}
m_{d} a_{p} k \\
f
\end{array}, \varepsilon \delta=a_{p} f k, \\
& \nu={ }_{m}^{a}, \varepsilon d=\zeta, \varepsilon d_{d}=\zeta_{d}, \varepsilon d_{i}=\zeta_{i}, \varepsilon \alpha=e
\end{aligned}
$$

Using the parameters in Eq. (17) in conjunction with Eqs (14) through (16), the following equations of motion are realized.

$$
\begin{aligned}
& q^{\prime \prime}+\varepsilon d q^{\prime}+\varepsilon \omega_{m} q-h u_{d}-\varepsilon h u_{i}=w^{2} \sin (w \tau) \\
& u_{d}^{\prime \prime}+\varepsilon\left(d_{d}+\alpha u_{d}^{2}\right) u_{d}^{\prime}-\varepsilon \delta q+u_{d}+\varepsilon u_{i}=0 \\
& u_{i}^{\prime \prime}+\varepsilon d_{i} u_{i}^{\prime}-\varepsilon \nu \delta q+\nu u_{d}+\varepsilon \nu u_{i}=0
\end{aligned}
$$

Notice that the nonlinear term in Eq. (19) $\left(\alpha u_{d}^{2}\right)$ is more simplified than originally expressed in Eq. (11). This term controls the restricted behavior of the decoupler and necessitates the use of perturbation techniques such as the averaging method to achieve an analytical solution. To utilize the averaging method to determine the frequency responses of the system governed by Eqs (18) through (20), the following solutions are assumed.

$$
\begin{aligned}
& q=r(\tau) \cos (w \tau+\phi(\tau)) \\
& u_{d}=r_{d}(\tau) \cos \left(w \tau+\phi_{d}(\tau)\right) \\
& u_{i}=r_{i}(\tau) \cos \left(w \tau+\phi_{i}(\tau)\right) \\
& q^{\prime}=-r(\tau) w \sin (w \tau+\phi(\tau)) \\
& u_{d}^{\prime}=-r_{d}(\tau) w \sin \left(w \tau+\phi_{d}(\tau)\right) \\
& u_{i}^{\prime}=-r_{i}(\tau) w \sin \left(w \tau+\phi_{i}(\tau)\right) \\
& q^{\prime \prime}=-w \sin (w \tau+\phi(\tau)) \begin{array}{c}
d r(\tau) \\
d \tau
\end{array}-r(\tau) w \cos (w \tau+\phi(\tau))\left(\begin{array}{c}
w \phi(\tau) \\
d \tau
\end{array}\right) \\
& u_{d}^{\prime \prime}=-w \sin \left(w \tau+\phi_{d}(\tau)\right) \begin{array}{c}
d r_{d}(\tau) \\
d \tau
\end{array} r_{d}(\tau) w \cos \left(w \tau+\phi_{d}(\tau)\right)\left(w+\begin{array}{c}
d \phi_{d}(\tau) \\
d \tau
\end{array}\right) \\
& u_{i}^{\prime \prime}=-w \sin \left(w \tau+\phi_{i}(\tau)\right) \begin{array}{c}
d r_{i}(\tau) \\
d \tau
\end{array}-r_{i}(\tau) w \cos \left(w \tau+\phi_{i}(\tau)\right)\left(\begin{array}{c}
d \phi_{i}(\tau) \\
d \tau
\end{array}\right)
\end{aligned}
$$

where:

$$
\begin{gathered}
\cos (w \tau+\phi(\tau)) \begin{array}{c}
d r(\tau) \\
d \tau
\end{array} \quad r(\tau) \sin (w \tau+\phi(\tau)) \begin{array}{c}
d \phi(\tau) \\
d \tau
\end{array}=0 \\
\cos \left(w \tau+\phi_{d}(\tau)\right) \begin{array}{c}
d r_{d}(\tau) \\
d \tau
\end{array}-r_{d}(\tau) \sin \left(w \tau+\phi_{d}(\tau)\right) \begin{array}{c}
d \phi_{d}(\tau) \\
d \tau
\end{array}=0
\end{gathered}
$$




$$
\cos \left(w \tau+\phi_{i}(\tau)\right) \begin{gathered}
d r_{i}(\tau) \\
d \tau
\end{gathered}-r_{i}(\tau) \sin \left(w \tau+\phi_{i}(\tau)\right) \begin{gathered}
d \phi_{i}(\tau) \\
d \tau
\end{gathered}=0
$$

Now Eqs (21) through (29) are substituted into Eqs (18) through (20) and utilized in conjunction with Eqs (30) through (32) to obtain expressions for the time derivatives of the amplitude and phase lag components of the solution (expressed in Appendix A). Next these solutions may be integrated over one period of oscillation $(T)$ as follows. Note these integrals must equal zero to obtain a steady state solution [7].

$$
\begin{aligned}
& 1 \int_{0}^{T} d r(\tau) d \psi=0 \\
& 1 \int_{0}^{T} r(\tau) d \phi(\tau) d \psi=0 \\
& d \tau \\
& T \\
& 1 \\
& T \int_{0}^{T} d r_{d}(\tau) d \psi_{d}=0 \\
& 1 \int_{0}^{T} r_{d}(\tau) d \phi_{d}(\tau) d \psi_{d}=0 \\
& 1 \int_{0}^{T} d r_{i}(\tau) d \psi_{i}=0 \\
& 1 \int_{0}^{T} r_{i}(\tau) d \phi_{i}(\tau) d \psi_{i}=0 \\
& T_{0}^{T} d \tau
\end{aligned}
$$

where:

$$
\begin{aligned}
& \psi=w \tau+\phi(\tau) \\
& \psi_{d}=w \tau+\phi_{d}(\tau) \\
& \psi_{i}=w \tau+\phi_{i}(\tau)
\end{aligned}
$$

The results of the integration in Eqs (33) and (34) are as follows.

$$
\begin{aligned}
& -{ }_{2}^{1}(\varepsilon d r+w \cos (\phi))=0 \\
& { }_{2 w}^{1}\left(\varepsilon \omega_{m} r-w^{2} r+w^{2} \sin (\phi)\right)=0
\end{aligned}
$$

Equations (39) and (40) may be used to obtain a frequency response function regarding the relative behavior of the engine mass. In addition, the frequency response of both the decoupler and inertia track are not of critical importance to the optimization process; therefore, if desired, the reader may investigate the behavior of the decoupler and inertia track across the frequency spectrum in the following references [3,6].

Utilizing Eqs (39) and (40) and solving for the cos and sin terms allows use of the trigonometric identity $\sin ^{2}+\cos ^{2}=1$ to arrive at the following frequency response function describing the relative engine mass displacement. 


$$
\left(\begin{array}{c}
r\left(\varepsilon \omega_{m}-w^{2}\right) \\
w^{2}
\end{array}\right)^{2}+\left(\begin{array}{c}
\varepsilon d r \\
w
\end{array}\right)^{2}=1
$$

Normally Eq. (41) would be solved for $w$ to obtain $w=f(r)$; however, for the purpose of this optimization process it becomes advantageous to determine $r=f(w)$ noting such a solution may readily be obtained.

$$
r(w)=\begin{gathered}
w^{2} \\
\sqrt{ }\left(w^{2}+\varepsilon^{2} d^{2}-2 \varepsilon \omega_{m}\right) w^{2}+\varepsilon^{2} \omega_{m}^{2}
\end{gathered}
$$

In order to optimize the engine mount, information regarding the absolute acceleration of the engine mass must be obtained. To achieve the frequency response function regarding the absolute acceleration, the relative displacement frequency response function must be transformed into the absolute displacement frequency response function noting that the first approximation to the solution of the relative motion equation is $q(\tau)=r(w) \cos (w \tau+\phi(\tau))$. Notice that a solution regarding the absolute displacement is sought in the form $u(\tau)=\beta(w) \cos (w \tau+\gamma(\tau))$. Also note that $q=u-\sin (w \tau)$; therefore, after using the appropriate trigonometric identities, the solution for the absolute displacement may be expressed as follows.

$$
\beta(w)=\begin{gathered}
\varepsilon \sqrt{ }\left(w^{2}-2 \omega_{m} \varepsilon+\varepsilon^{2} \omega_{m}+\varepsilon^{2} d^{2}\right)\left(\omega_{m}^{2}+d^{2} w^{2}\right) \\
\left(w^{2}-2 \omega_{m} \varepsilon+\varepsilon^{2} \omega_{m}+\varepsilon^{2} d^{2}\right) w
\end{gathered}
$$

Utilizing Eq. (43), the absolute acceleration frequency response function may be defined as follows.

$$
A(w)=w^{2} \beta(w)=\begin{gathered}
\varepsilon w \sqrt{ }\left(w^{2}-2 \omega_{m} \varepsilon+\varepsilon^{2} \omega_{m}+\varepsilon^{2} d^{2}\right)\left(\omega_{m}^{2}+d^{2} w^{2}\right) \\
\left(w^{2}-2 \omega_{m} \varepsilon+\varepsilon^{2} \omega_{m}+\varepsilon^{2} d^{2}\right)
\end{gathered}
$$

\subsection{Optimization process}

The determination of an optimum design for a set of constraints is dependent upon the choice of cost functions. For this analysis the cost functions are functions of both the absolute acceleration and relative displacement frequency responses. More specifically, the cost function is defined by the root mean square (RMS) value of the absolute acceleration and relative displacement as follows [10].

$$
\begin{aligned}
& R=\sqrt{1} \begin{array}{c}
1 \\
w_{2}-w_{1}
\end{array} \int_{w_{1}}^{w_{2}}(A(w))^{2} d w
\end{aligned}
$$

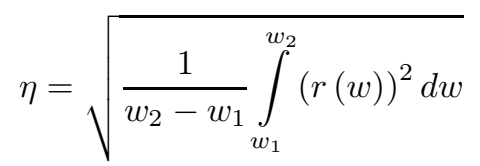

Noting that numerical integration of Eqs (45) and (46) yields a three-dimensional surface of possible values of $\omega_{m}$ and $d$ by which to find an appropriate combination to use as parameters defining the hydraulic mount it becomes advantageous to seek an expression to define a minimum relationship between $R$ and $\eta$. Note that the RMS solutions from Eqs (45) and (46) result in a large number of possible mount configurations which may not necessarily be optimal solutions. In addition, the optimization analysis requires a minimum of absolute acceleration for a specific relative displacement; therefore, a relationship between Eqs (45) and (46) must be found. More specifically, it becomes advantageous to find the first derivative of $R$ with respect to $\eta$. However, Eqs (45) and (46) show there is no direct relationship between these variables; therefore, the following use of derivatives is employed to obtain such a relationship.

$$
\frac{\partial R}{\partial \eta}=\frac{\partial R \partial d}{\partial d \partial \eta}=\begin{gathered}
R_{I J, d} \\
\eta_{I J, d}
\end{gathered}=R_{I J, \eta}=0
$$

Equation (47) gives the necessary relationship to find a minimum between $R$ and $\eta$; however, there is no guarantee that this solution ever exactly equals zero due to the numerical integration in Eqs (45) and (46) and the numerical 
differentiation then required by Eq. (47). However, it does become obvious that for a local minimum to exist there will be a change of signs of the derivative near the location of the minimum; therefore, a linear interpolation between said points will be utilized to approximate the location of the minimum. Before the interpolation process may be implemented a method of numerically differentiating Eq. (47) must be determined. There are many methods for numerical differentiation available and discussions on these methods may be found in any numerical analysis text; however, for this analysis a central difference approximation will be utilized and expressed in indicial notation as follows.

$$
R_{I J, d}=\begin{gathered}
1 \\
2 H
\end{gathered}\left(R_{I, J+1}-R_{I, J-1}\right), \eta_{I J, d}=\begin{gathered}
1 \\
2 H
\end{gathered}\left(\eta_{I, J+1}-\eta_{I, J-1}\right)
$$

The differentiation process outlined in Eq. (48) is a standard central difference approximation where $H$ is equivalent to the step size in $d[8,9]$. Now that the derivatives are obtained the local minimum can be interpolated as follows.

$$
R_{o p t}=\begin{aligned}
& R_{I J, \eta}^{-}\left(R^{-}-R^{+}\right) \\
& R_{I J, \eta}^{+}-R_{I J, \eta}^{-}
\end{aligned}+R^{-}, \eta_{o p t}=\begin{aligned}
& R_{I J, \eta}^{-}\left(\eta^{-}-\eta^{+}\right) \\
& R_{I J, \eta}^{+}-R_{I J, \eta}^{-}
\end{aligned}+\eta^{-}
$$

With the optimum line defined it becomes important to determine the relationships between $d$ and $\omega_{m}$ to actual geometric properties of the mount. More specifically, using Eqs (13) and (17) the following relationships between the inertia track and decoupler are found.

$$
\begin{aligned}
& A_{i}=\begin{array}{c}
C_{1} B_{r}^{2} A_{d} \omega_{m} \\
M d^{2}\left(K_{r} C_{1}+A_{p}^{2}\right)
\end{array} \\
& \Delta=\begin{array}{c}
K A_{d}\left(B_{r} C_{1} \omega_{m}\right)^{2}-\rho d^{2} t_{d}\left(A_{p}^{4}+2 A_{p}^{2} C_{1} K_{r}+C_{1}^{2} K_{r}^{2}\right) \\
2 \rho d^{2}\left(K_{r} C_{1}+A_{p}^{2}\right)^{2}
\end{array}
\end{aligned}
$$

Equations (50) and (51) illustrate the required decoupler gap size and inertia track area as a function of $\omega_{m}$ and $d$ where $t_{d}$ is the thickness of the decoupler disk noting that the length of the decoupler is $2 \Delta+t_{d}$.

\subsection{Numerical analysis}

As an additional check on the nonlinear analysis, a numerical analysis was utilized. However, the numerical analysis provides its own set of unique challenges. For example, in order to achieve a frequency response the equations of motion must be solved for a certain time domain sufficient enough to obtain a steady state response. This process is repeated at several frequency values until the entire frequency range of interest is explored. The difficulty enters in at the determination of the steady state solution. More specifically, when does the numerical time domain solution achieve a steady state condition? In many algorithms, the steady state solution is obtained by solving the time domain solution for several oscillations of the solution and then the amplitude of the solution is obtained. Next, the solution is obtained for a larger number of oscillations, and the amplitude is taken and compared to the previous solution. If the two solutions match within a reasonable tolerance, the solution is considered to be steady state in nature. While this method is effective, it is not very efficient from a computational and mathematical perspective. This investigation utilizes a new method based on the energy-rate method proposed by Jazar [11]. This method finds the total energy of the system, differentiates it with respect to time to achieve a rate of change of the total energy, and then the solution is integrated over one period of oscillation. If this integral equals zero the solution is behaving in a steady state manner.

To apply this method to the nonlinear model of the hydraulic engine mount a description of the rate of change of energy must be found. Such an equation may be described in the following format [11].

$$
\dot{E}=-\dot{x} \cdot g(x, \dot{x}, t)
$$

where:

$$
\ddot{x}+f(x)=-g(x, \dot{x}, t)
$$

To apply Eq. (52) to the hydraulic engine mount system, Eqs (18) through (20) may be written as follows. 


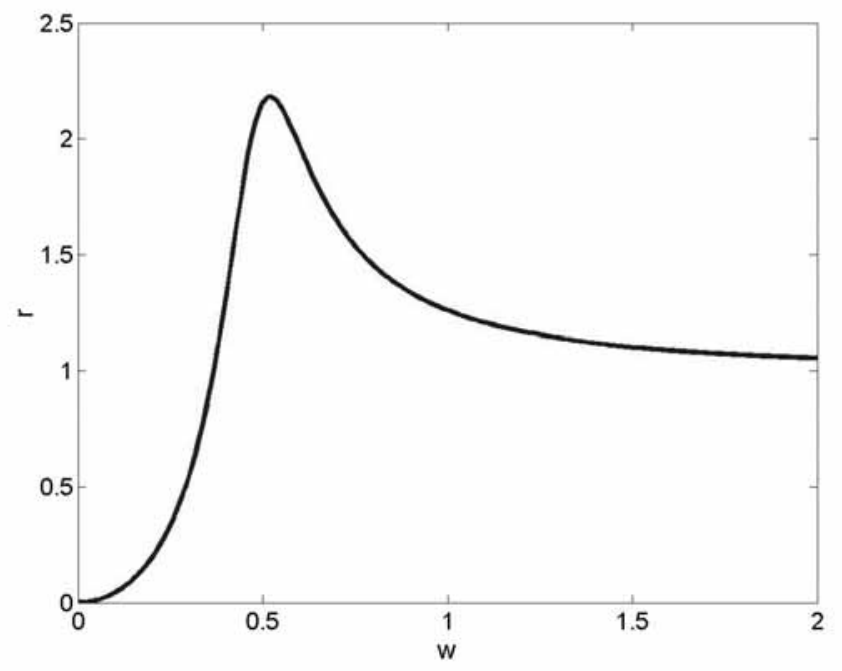

Fig. 4. Relative engine mass displacement.

$$
\begin{aligned}
& \dot{E}=q^{\prime}\left(w^{2} \sin (w \tau)-\left(\varepsilon d q^{\prime}-h u_{d}-\varepsilon h u_{i}\right)\right) \\
& \dot{E}_{d}=-u_{d}^{\prime}\left(\varepsilon\left(d_{d}+\alpha u_{d}^{n}\right) u_{d}^{\prime}-\varepsilon \delta q+\varepsilon u_{i}\right) \\
& \dot{E}_{i}=-u_{i}^{\prime}\left(\varepsilon d_{i} u_{i}^{\prime}-\varepsilon \nu \delta q+\nu u_{d}\right)
\end{aligned}
$$

Equations (53) through (55) may now be integrated numerically over one period of oscillation (T) as follows.

$$
\begin{aligned}
& E_{a v}=\frac{1}{T} \int_{0}^{T}(\dot{E}) d t \\
& E_{a v d}=\frac{1}{T} \int_{0}^{T}\left(\dot{E}_{d}\right) d t \\
& E_{a v i}=\frac{1}{T} \int_{0}^{T}\left(\dot{E}_{i}\right) d t
\end{aligned}
$$

Equations (56) through (58) are utilized to determine if the system is at a steady state condition. If these equations do not equal zero, the numerical solution is obtained for another period of motion. This process is repeated until the Eqs (56) through (58) approach zero within a reasonably small tolerance.

\section{Results}

The frequency response functions of the system supported by a hydraulic mount are of critical importance to the optimization process; therefore, the validity of the frequency response functions is checked on an existing model of a hydraulic engine mount (values for this mount are given in Table 1). Figure 4 illustrates the nondimensional behavior of the relative engine mass displacement, Fig. 5 illustrates the absolute displacement of the engine mass, and Fig. 6 illustrates the absolute acceleration of the engine mass. As is readily apparent in Fig. 4, the system 


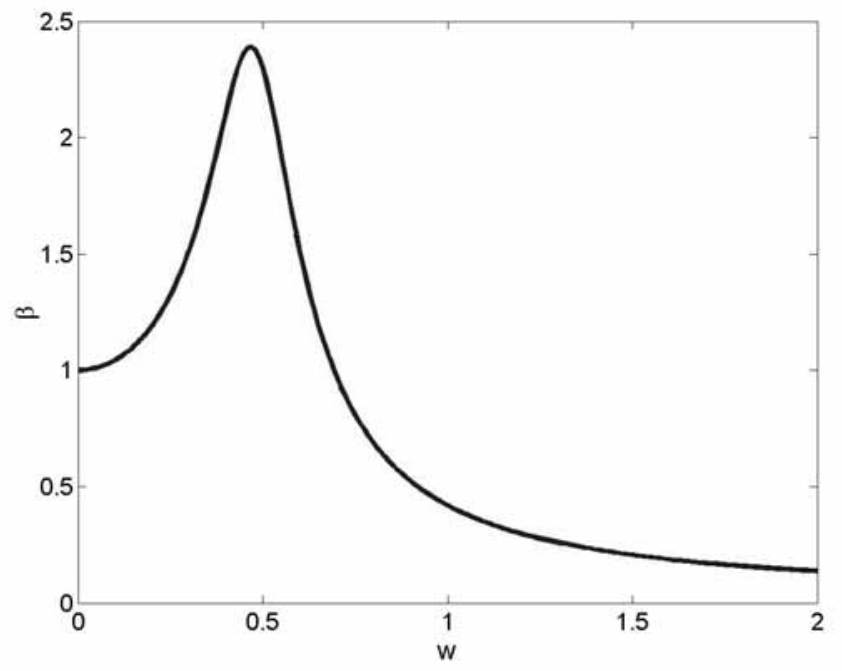

Fig. 5. Absolute engine mass displacement.

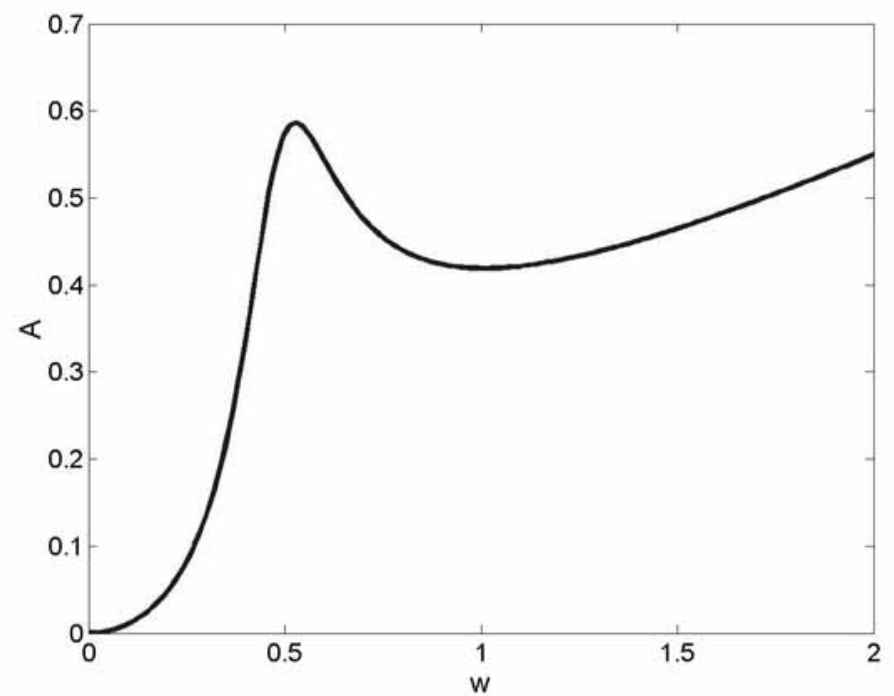

Fig. 6. Absolute acceleration of the engine mass.

behaves in a manner similar to a linear system even though the hydraulic engine mount is inherently nonlinear. However, this is not totally unexpected noting that the primary spring of the system, the upper rubber structure of the mount, is modeled as a linear spring. In addition, a large portion of the damping of the mount comes directly from the hysteresis inherent in the upper rubber structure. As with the stiffness of the upper rubber, the damping was modeled as an equivalent linear viscous damper. Therefore, noting the prevalence of such factors, the appearance of linear-like behavior is not surprising.

Figures 7 through 9 illustrate the frequency responses of the system as a comparison between numerical solutions and the solutions obtained via the averaging method. As is readily apparent, the solutions match almost identically throughout the entire frequency spectrum analyzed thereby verifying the averaging method solution.

Before undertaking the optimization analysis, it becomes advantageous to note the sensitivity of the frequency 
Table 1

Numerical values for the baseline hydraulic mount

$\begin{array}{ccc}\text { Symbol } & \text { Value } & \text { Dimension } \\ A_{d} & 5.303 \mathrm{E}-05 & \mathrm{~m}^{2} \\ A_{i} & 4.297 \mathrm{E}-05 & \mathrm{~m}^{2} \\ A_{p} & 0.007 & \mathrm{~m}^{2} \\ B_{r} & 2000 & \mathrm{~N}^{*} \mathrm{~s} / \mathrm{m} \\ B_{d} & 0.103 & \mathrm{~N}^{*} \mathrm{~s} / \mathrm{m} \\ B_{i} & 2.409 & \mathrm{~N}^{*} \mathrm{~s} / \mathrm{m} \\ C_{1} & 4.08 \mathrm{E}-10 & \mathrm{~m}^{5} / \mathrm{N} \\ C_{2} & 4.00 \mathrm{E}-08 & \mathrm{~m}^{5} / \mathrm{N} \\ \Delta & 3.18 \mathrm{E}-04 & \mathrm{~m} \\ K_{r} & 120000 & \mathrm{~N} / \mathrm{m} \\ M & 75 & \mathrm{~kg} \\ M_{d} & 5.220 \mathrm{E}-04 & \mathrm{~kg} \\ M_{i} & 9.887 \mathrm{E}-03 & \mathrm{~kg} \\ Y & 1.000 \mathrm{E}-03 & \mathrm{~m} \\ \rho & 912 & \mathrm{~kg} / \mathrm{m}^{3} \\ \mu & 0.380 & \text { Nm } / \mathrm{s} \\ E & 5.000 \mathrm{E}-02 & \text { dimensionless } \\ \varepsilon & 8.104 \mathrm{E}-01 & \text { dimensionless } \\ h & 1.444 \mathrm{E}-04 & \text { dimensionless } \\ \delta & 1.016 \mathrm{E}+03 & \text { dimensionless } \\ \nu & 4.279 \mathrm{E}-02 & \text { dimensionless } \\ d_{d} & 2.111 \mathrm{E}+00 & \text { dimensionless } \\ d_{i} & 2.605 \mathrm{E}+00 & \text { dimensionless } \\ \alpha & 1.024 \mathrm{E}+00 & \text { dimensionless }\end{array}$

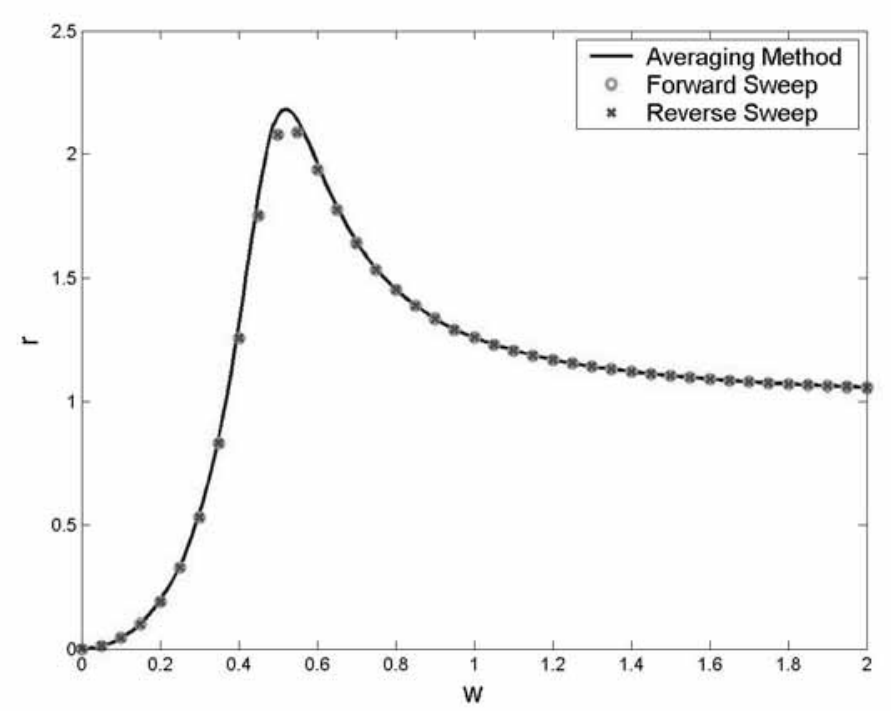

Fig. 7. Relative engine mass displacement.

response solutions to changes of parameter values; therefore, Figs 10 through 15 illustrate the effects of altering the damping and stiffness terms of the engine mass equation to the relative and absolute displacements in conjunction with the absolute acceleration.

Figure 10 illustrates the relative engine mass displacement and its response to changing the damping value associated with the mount. As is typical with any vibration isolation system, increasing damping decreases the amplitude in the domain of primary resonance. This behavior is again noted in Fig. 11, which displays the absolute engine mass amplitude. However, in this figure, it becomes apparent that under higher frequency excitations, the 


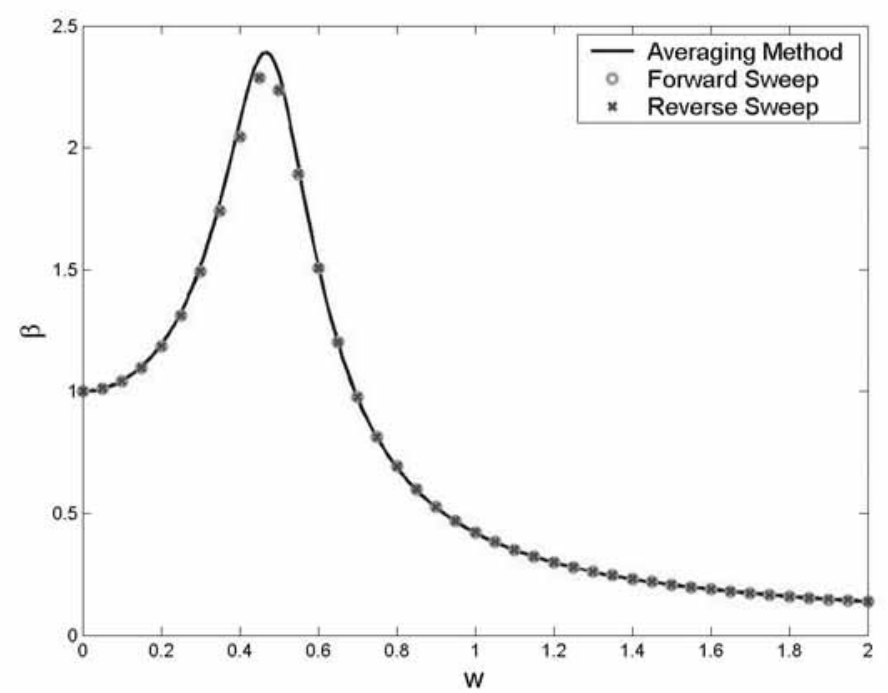

Fig. 8. Absolute engine mass displacement.

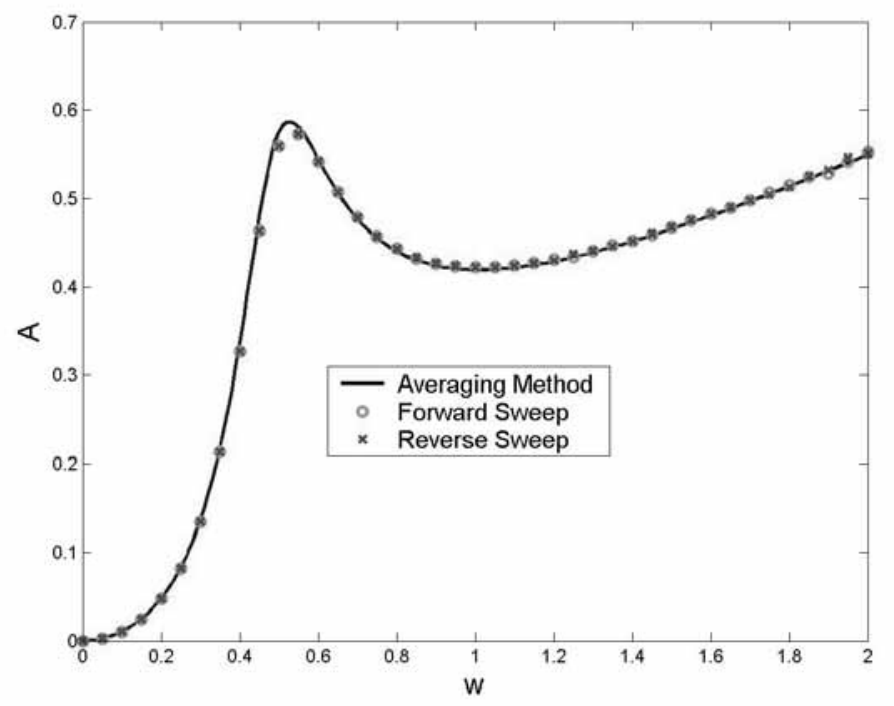

Fig. 9. Absolute engine mass acceleration

amplitude increases as the damping term increases in magnitude. Again this is typical of vibration isolators.

Figure 12 illustrates the response of the absolute acceleration to the effects of changing damping values. Again, the behavior appears typical of most vibration isolators. However, here the effects of changing the damping become more obvious than in Figs 10 and 11. Here it may be shown that by altering $d$, the cross-sectional area of the inertia track is being altered. Looking at the definition of $d$ given in Eqs (13) and (17), it may be seen that by increasing the value of $A_{i}$ actually decreases the value of $d$; however, if the value of $A_{i}$ is decreased, the value of $d$ increases. More specifically, this says that the inertia track geometry becomes very critical to the overall damping of the mount, especially noting that $B_{r}$, the equivalent damping of the upper rubber structure, is very difficult to alter in any appreciable manner. 


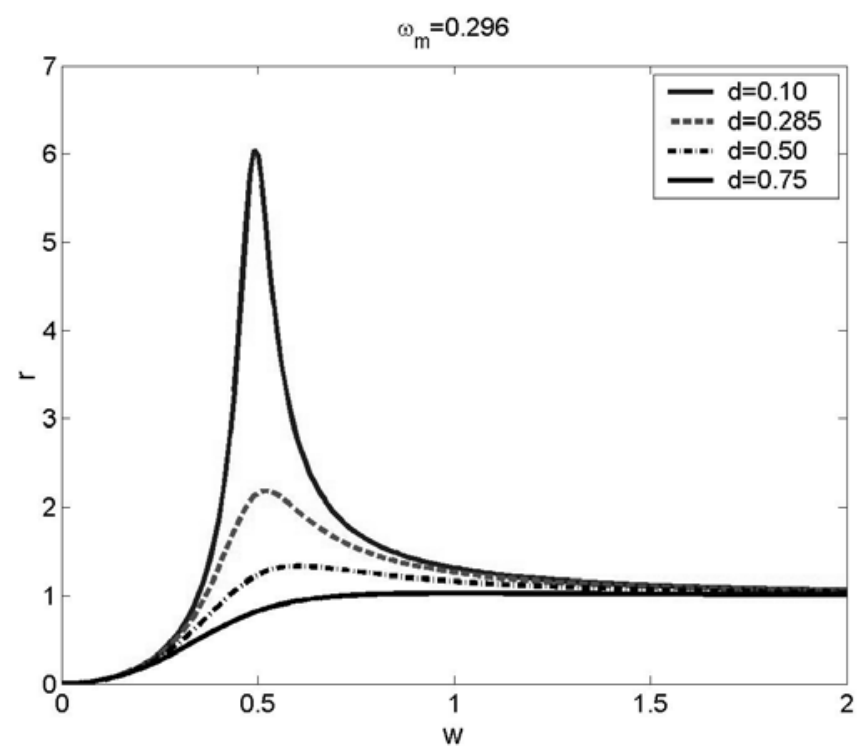

Fig. 10. Relative engine mass displacement.

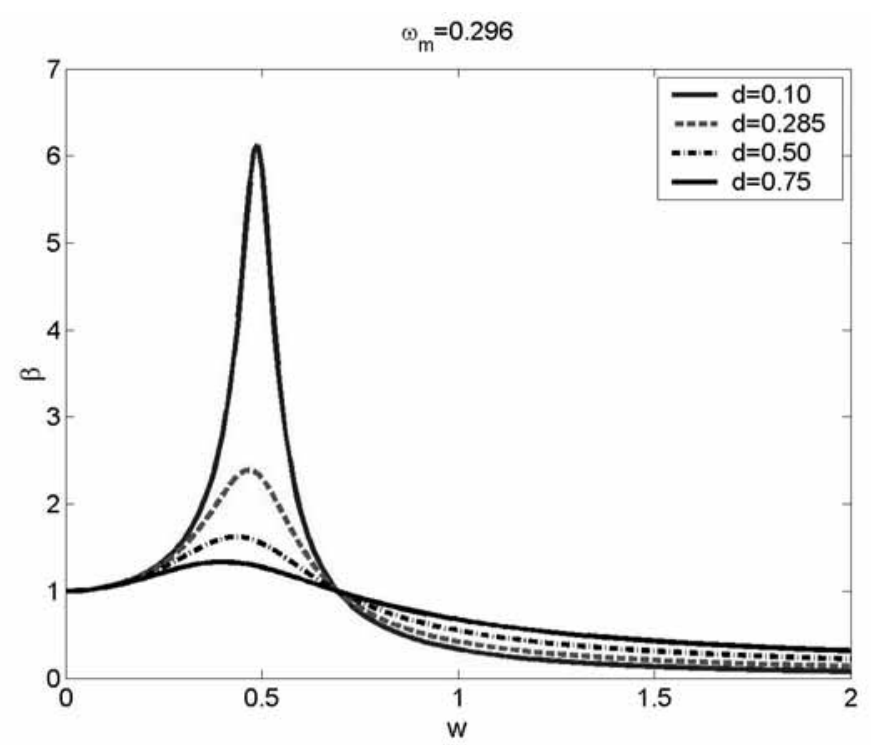

Fig. 11. Absolute engine mass displacement.

Figures 13 through 15 illustrate the behavior of the engine mount while holding the damping value constant and varying the stiffness value of the mount. Figure 13 illustrates the relative displacement of the engine mass, Fig. 14 illustrates the absolute displacement of the engine mass, and Fig. 15 illustrates the absolute acceleration of the engine mass. As can be readily noted, changing the stiffness terms directly influences the resonant frequency of the mount. In addition, if Fig. 15 is examined, the transmissibility of the mount is greatly altered by small changes in the stiffness of the mount. More specifically, if the parameters in Eqs (13) and (17) are analyzed, the decoupler properties play a critical role in determining the resonant frequency. More specifically, the decoupler area and mass, which is a function of the length of the decoupler and hence the gap size greatly effect the location of the resonant frequency, and hence the switching point between high and low damping modes. Therefore, by altering the decoupler length, 


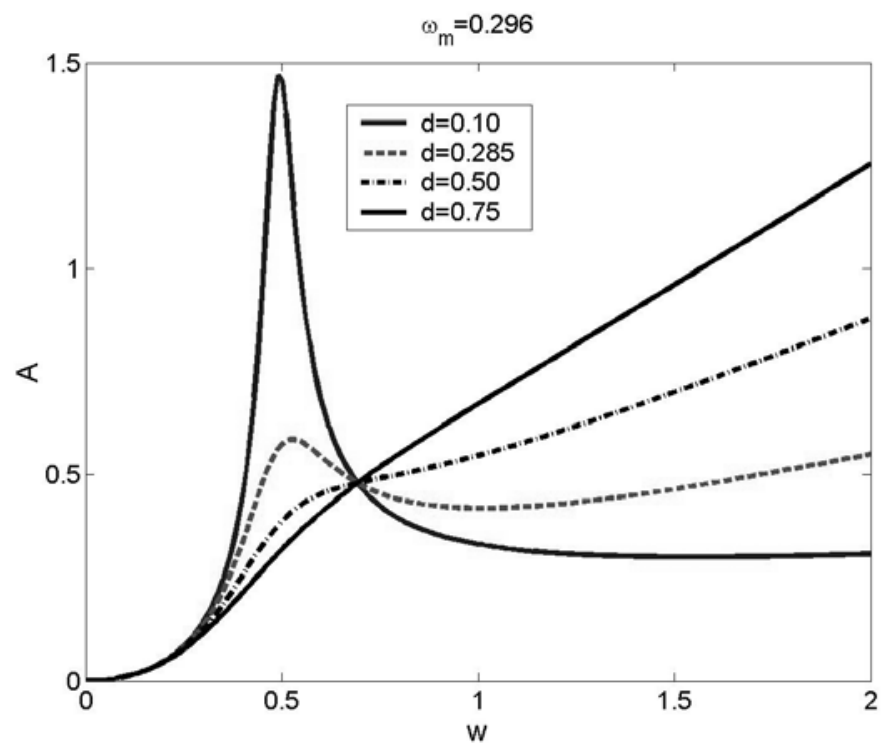

Fig. 12. Absolute engine mass acceleration.

the mass of the fluid in the decoupler is affected and the stiffness of the mount is affected. It becomes apparent in Figs 13 through 15 that by altering said properties, the overall behavior of the engine mount is greatly affected. Most notably, if a larger gap size is used, the mass of the fluid in the decoupler increases and the decoupler resonant frequency will occur earlier. However, the primary resonant domain of the mount occurs later in the frequency spectrum due to the increase in the overall mount stiffness $\left(\omega_{m}\right)$. In addition, Figs 13 through 15 illustrate that as $\omega_{m}$ increases so does the peak amplitude. More specifically, because the decoupler gap size is increasing, the switching point between high and low damping modes occurs earlier in the frequency spectrum, and because the mount is becoming less damped the peak amplitude increases accordingly. Such logic becomes advantageous when trying to determine the optimal location of the switching point for the mount. The switching point is the point in which the decoupler changes from large amplitudes, closing of the decoupler, to small amplitudes, the decoupler remains open. This point greatly affects the transmissibility of the mount as is readily apparent in Fig. 15. More specifically, if the primary resonant frequency is shifted upward, smaller decoupler gap size, the acceleration transmissibility becomes lower in magnitude than if the decoupler gap size is increased. In addition, this also allows the mount to provide lower relative and absolute motion of the engine mass as is apparent in Figs 13 and 14. However, the stiffness of the mount is not solely dependent upon the decoupler properties; it also is a function of the overall stiffness of the engine mount, the fluid density, and the properties of the inertia track. The appearance of these other factors in the value of the stiffness, hence primary resonant frequency, of the engine mount introduces difficulty in the determination of an appropriate method for optimization. It becomes apparent that some sort of cost function must be utilized to determine the optimal method for determining the decoupler and inertia track geometry in conjunction with choosing an appropriate fluid, and in choosing an appropriate stiffness value of the upper rubber structure. Therefore, Eqs (45) and (46) are utilized as cost functions to weigh the importance of these variables across the frequency spectrum of interest, which for the purposes of this analysis is below $30 \mathrm{~Hz}$.

Figure 16 illustrates the multidimensional surface produced by Eqs (45) and (46) projected to the $R-\eta$ plane to make a useable design chart by which the hydraulic engine mount may be optimized. Figures 17 and 18 show Fig. 16 broken into its respective components. Figure 17 illustrates the effects of keeping the system damping constant while varying the stiffness of the system. Where as Fig. 18 illustrate the effects of keeping the stiffness of the system constant and varying the damping value. Overlaying Figs 17 and 18, Fig. 16 may be obtained. Figure 16 may be utilized to obtain information regarding the ideal damping and stiffness values for the mount while operating within a certain displacement constraint and keeping acceleration to a minimum. More specifically, if any one variable is imposed upon the system as a constraint it becomes possible to find the optimum system parameters to minimize the remaining variable. 


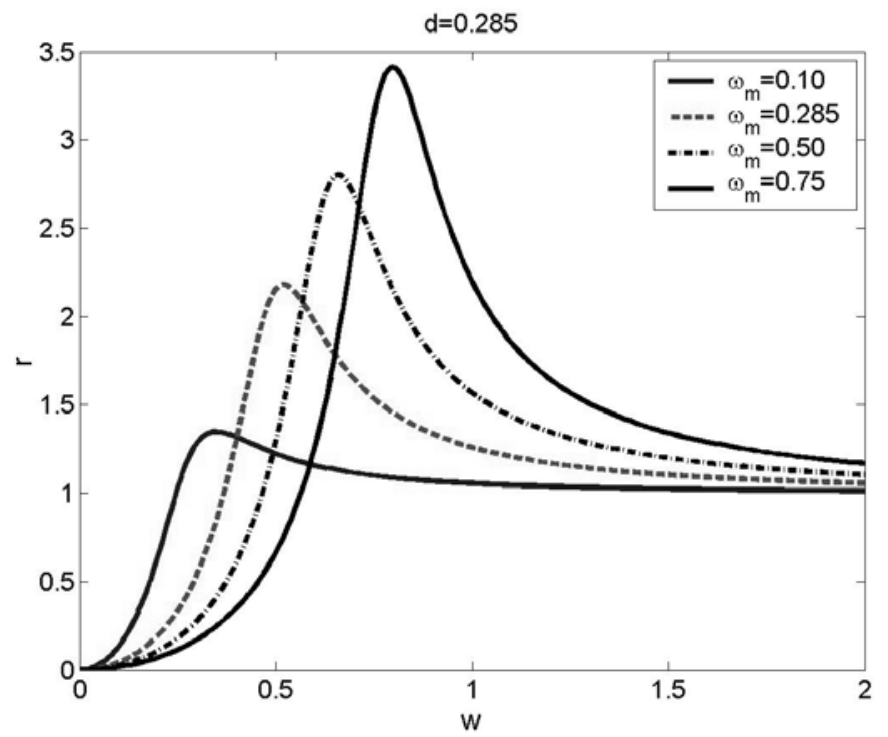

Fig. 13. Relative engine mass displacement.

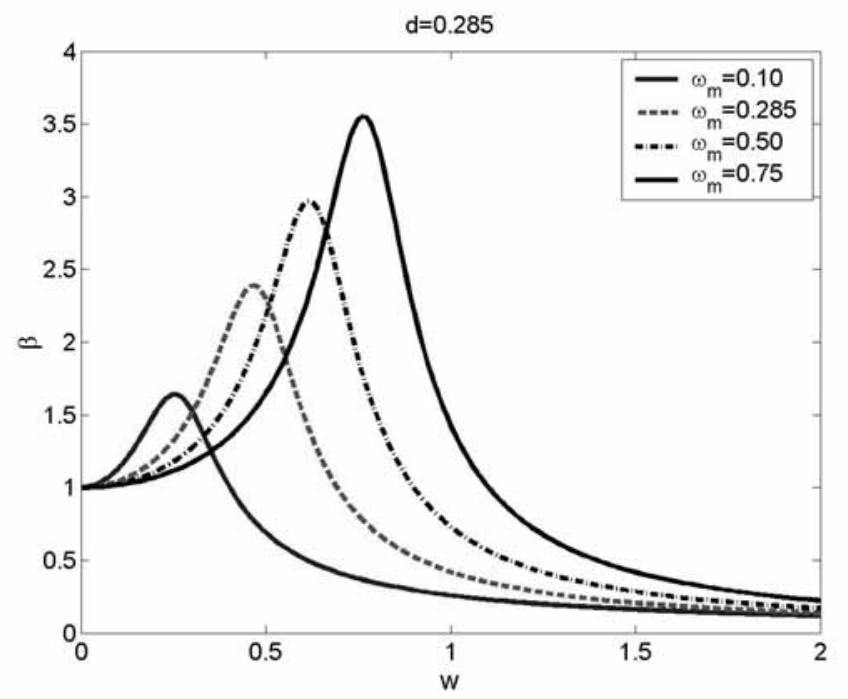

Fig. 14. Absolute engine mass displacement.

Figure 19 illustrates the existence of a line of minima between the RMS of the acceleration and relative displacement transmissibility's. More specifically, Fig. 19 illustrates the line of minima to exist at the intersection of two surfaces. One of these surfaces is a plane at the location of $R_{I J, \eta}$ is equal to zero, and the other surface represents the results of the numerical differentiation in Eq. (47) plotted against $d$ and $\omega_{m}$. With the line of minima defined it may be noted that any point on this line yields the appropriate damping and stiffness values for the hydraulic mount if relative displacement is known as a constraint and acceleration is desired to be at a minimum. Figure 20 illustrates the line of minima projected onto the $R-\eta$ plane from Fig. 16 to make this line a useable feature of the design chart. Figure 21 is a magnification of Fig. 20 in the low acceleration, high amplitude region of the chart to better illustrate the line of minima. In addition, Fig. 21 provides a more useable design chart for designs that may be concerned with said region (see Appendix B for a table of values defining the line of minima). 


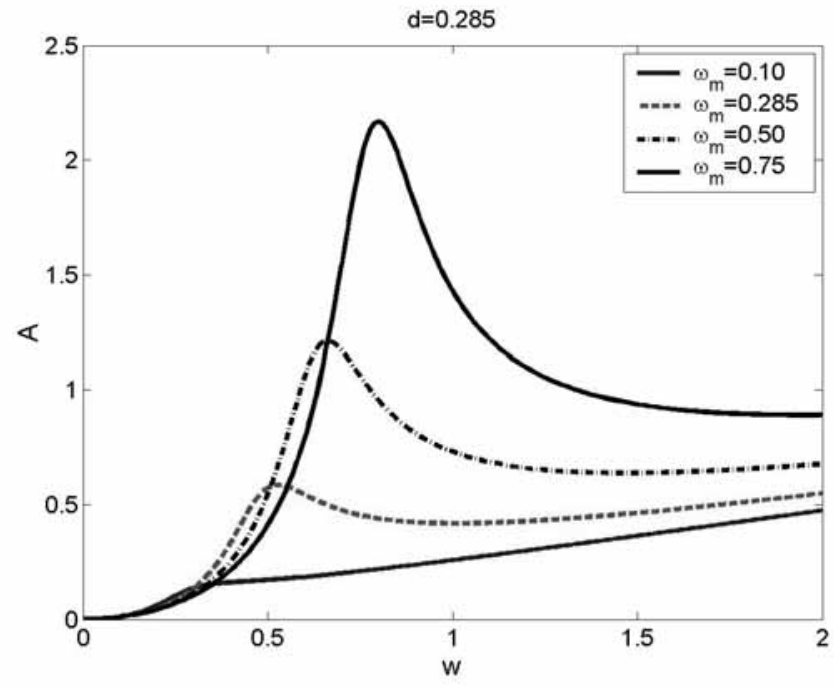

Fig. 15. Absolute engine mass acceleration.

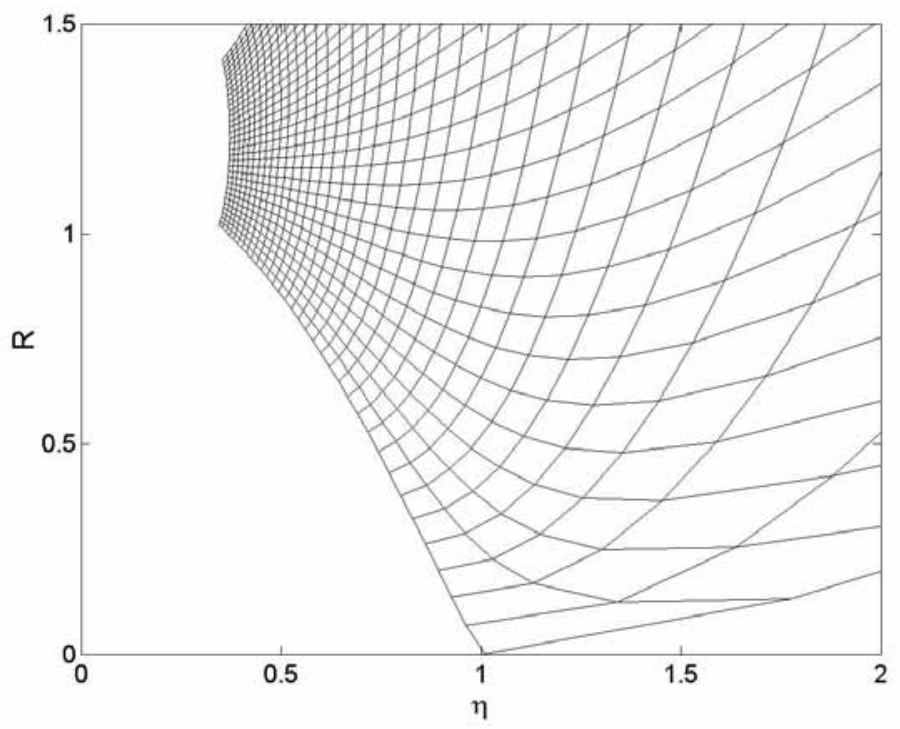

Fig. 16. Two-dimensional projection of the RMS surface to the $R-\eta$ plane.

Figures 22 and 23 more clearly illustrate the results of Eqs (45) and (46) in conjunction with showing the line of minima whereas Fig. 24 illustrates said line of minima in the $\omega_{m}-d$ plane. By observing the line of minima along the surfaces, the optimum relationship between $R$ and $\eta$ is more clearly illustrated as it varies with respect to either $\omega_{m}$ or $d$ respectively.

Figures 25 through 27 illustrate the effects of the optimization analysis on the frequency response of the system. Four configurations are considered by random selection as follows: point $1\left(d=0.201, \omega_{m}=0.26369\right)$, point 2 $\left(d=0.701, \omega_{m}=0.77793\right)$, point $3\left(d=0.201, \omega_{m}=0.5760\right)$, and point $4\left(d=0.101, \omega_{m}=0.26369\right)$. Notice that two points exist on the line of minima, points 1 and 2, whereas points 3 and 4 are projections of point 1 away from the line of minima while holding the damping and stiffness constant respectively.

Figures 25 and 26 illustrate the effects of the optimization process upon the relative and absolute amplitude of 


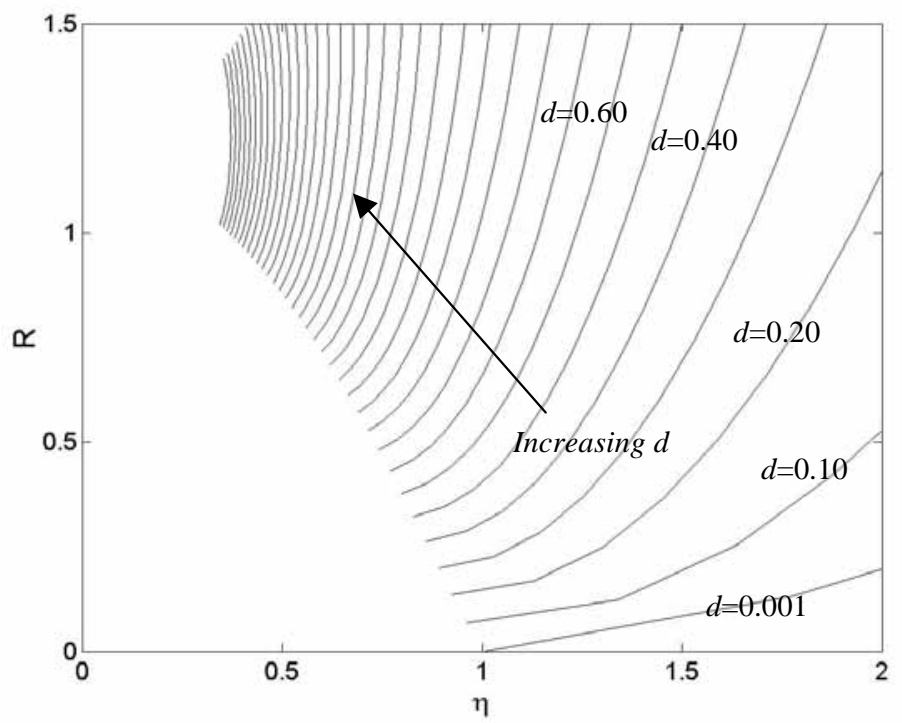

Fig. 17. Lines of constant damping with varying stiffness.

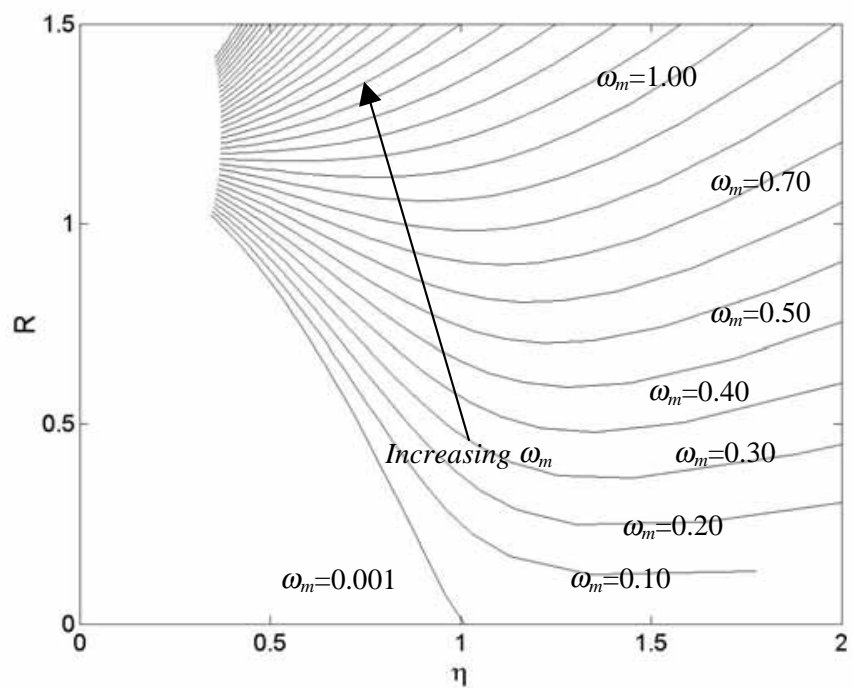

Fig. 18. Lines of constant stiffness with varying damping.

the engine mass respectively. Notice that the two points, points 1 and 2 , illustrate the lowest amplitude as can be expected noting these points exist on the line of minima, where as the frequency responses arising from points 3 and 4 exhibit substantially higher resonant peaks as compared to points 1 and 2 . This however is easily explained noting that points 3 and 4 lie substantially far from the line of minima and therefore must not exhibit an optimum frequency response.

Figures 25 and 26 also illustrate that from a displacement constraint perspective, point 2 is by far the superior candidate in comparison to the remaining points. However, point 1 also exhibits relatively low amplitude near resonance when compared to the frequency responses of both points 3 and 4 . However, to verify if point 2 is an ideal model it becomes desirable to investigate the acceleration transmissibility of the four points. 


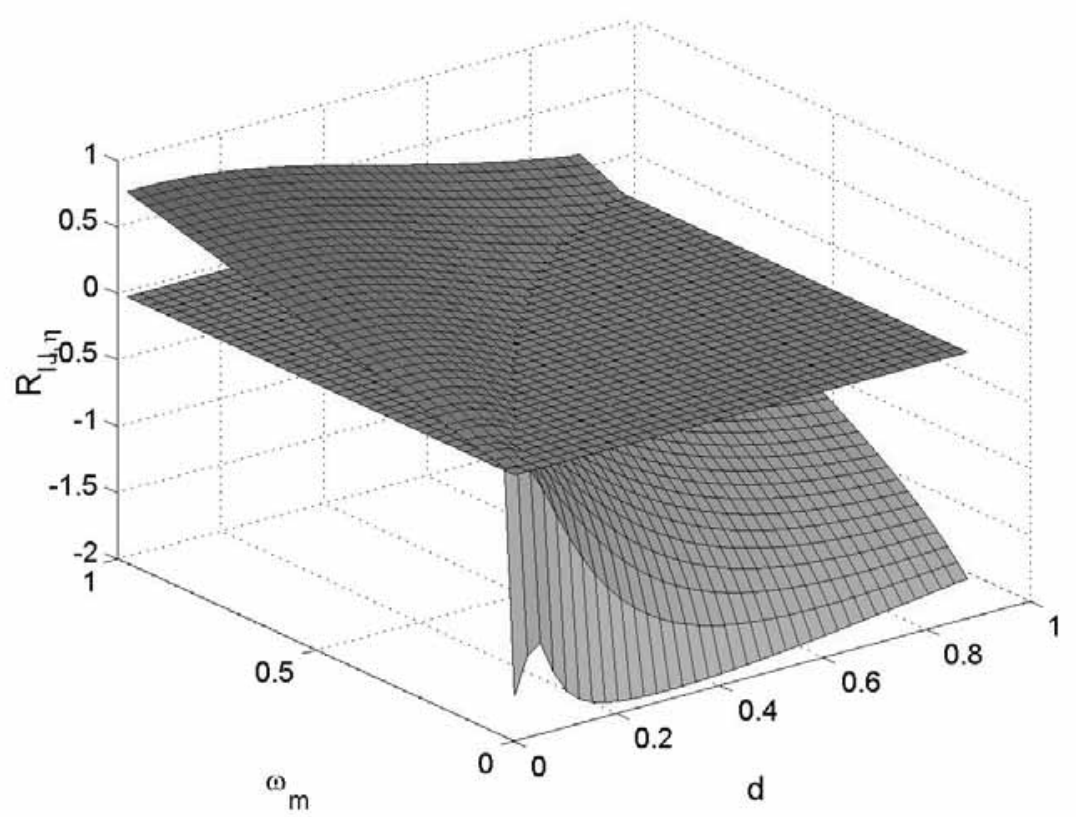

Fig. 19. Definition of the line of minima.

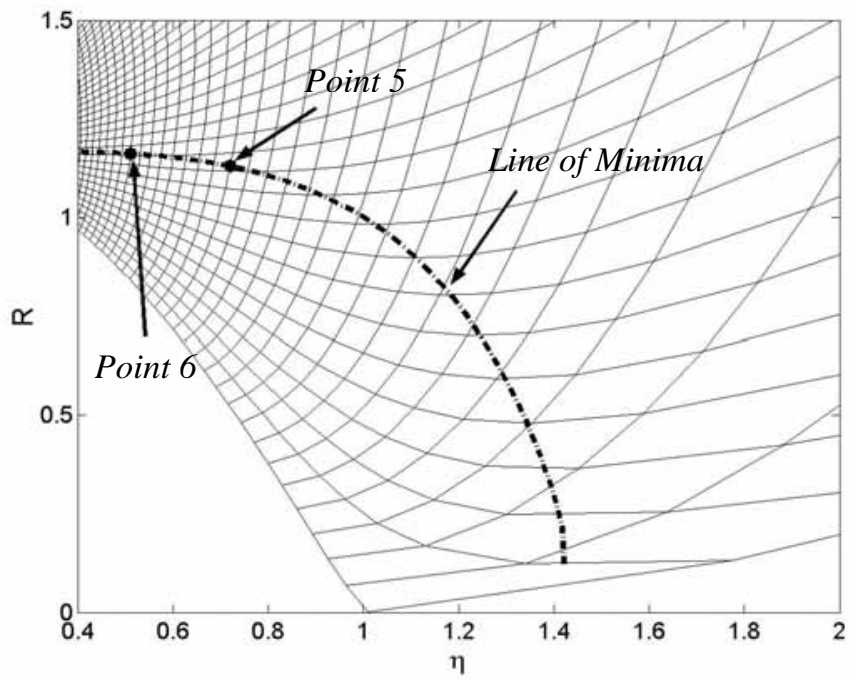

Fig. 20. $R-\eta$ plane illustrating the line of minima.

Figure 27 illustrates the acceleration transmissibility of the four configurations. Here it may be seen that again points 1 and 2 illustrate better behavior than points 3 and 4 in the low frequency spectrum below resonance. However, as frequency increases, point 2 exhibits a relatively high transmissibility as compared to the other three points. This figure shows that if minimized acceleration transmissibility is the goal of the design, as is often the case, that point 1 is the optimal design noting its relatively low acceleration transmissibility and acceptable displacement transmissibility. 


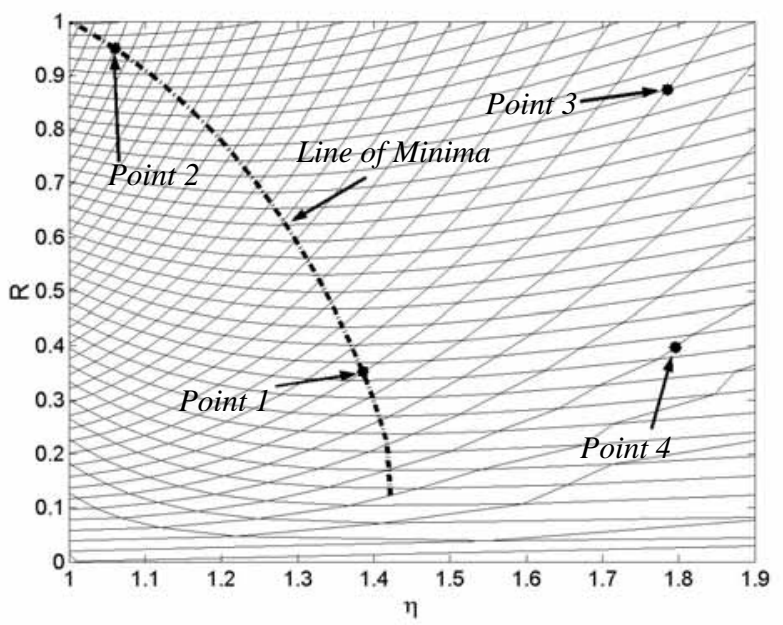

Fig. 21. Magnified view of the $R-\eta$ plane illustrating the line of minima.

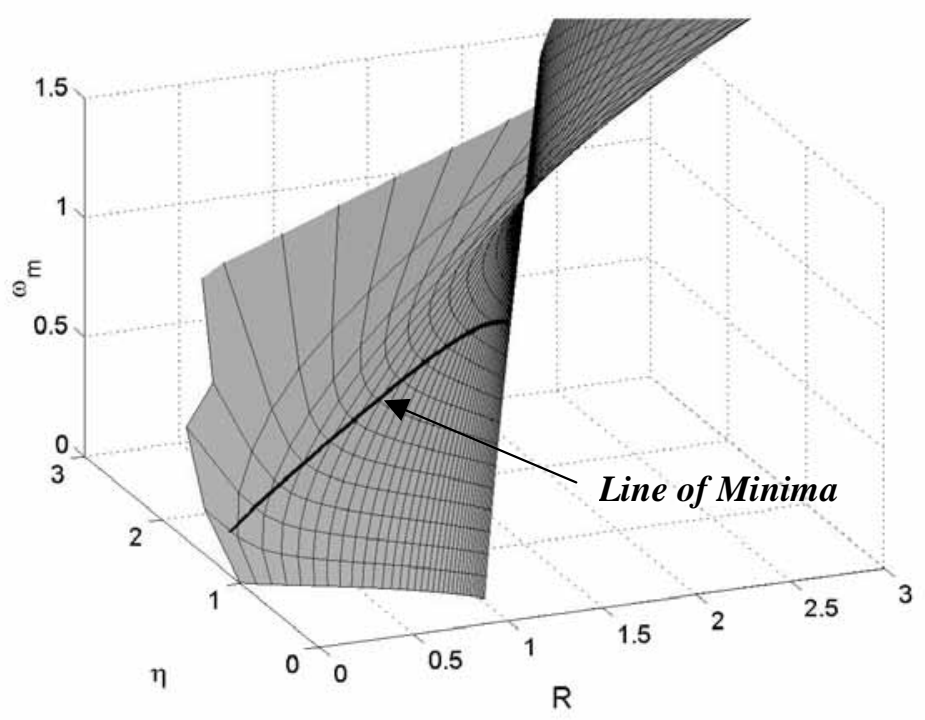

Fig. 22. RMS values of acceleration and relative displacement compared to wn illustrating the line of minima.

Figures 25 through 27 illustrate the effects of selecting a point on the line of minima and selecting a point away from the line. However, these figures do little in comparing several points on the line of minima. More specifically, what does the tradeoff between relative displacement and absolute acceleration illustrated by the line of minima mean in the frequency domain? Figs 28 through 30 illustrate this meaning by selecting several points that exist on the line of minima and comparing their frequency responses. The selected points are as follows: point $1(d=0.201$, $\left.\omega_{m}=0.26369\right)$, point $2\left(d=0.701, \omega_{m}=0.77793\right)$, point $5\left(d=0.951, \omega_{m}=0.90982\right)$, and point $6(d=2.001$, $\left.\omega_{m}=1.0986\right)$.

Figures 28 and 29 illustrate the relative displacement and absolute displacement frequency responses respectively. These figures illustrate that as the value of damping increases along the line of minima, hence stiffness, the displacement transmissibility is greatly reduced. However, if the absolute acceleration illustrated in Fig. 30 is considered, the results of increasing the damping along the line of minima show an increase in acceleration transmissibility. 


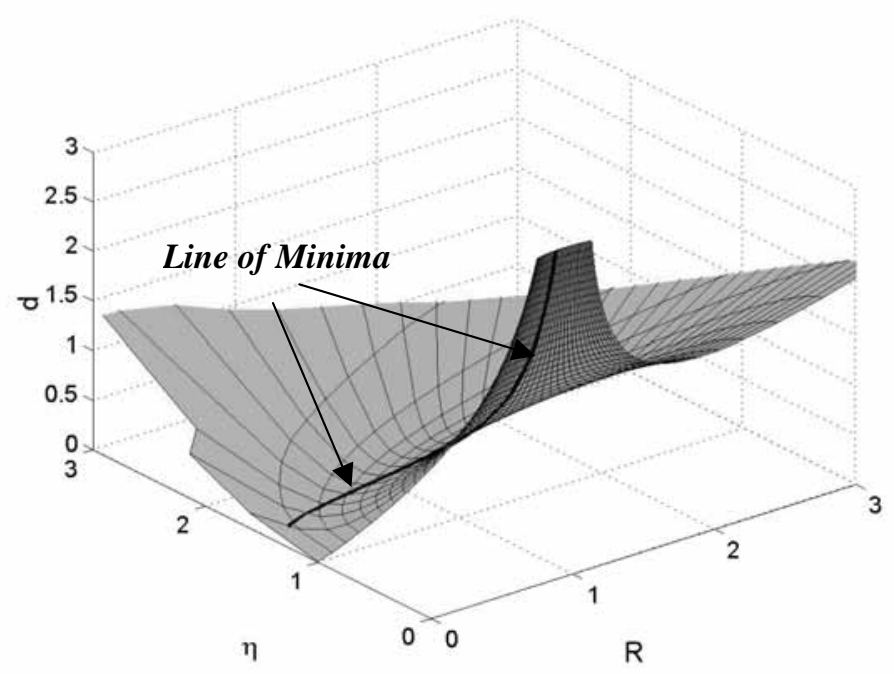

Fig. 23. RMS values of acceleration and relative displacement compared to $d$ illustrating the line of minima.

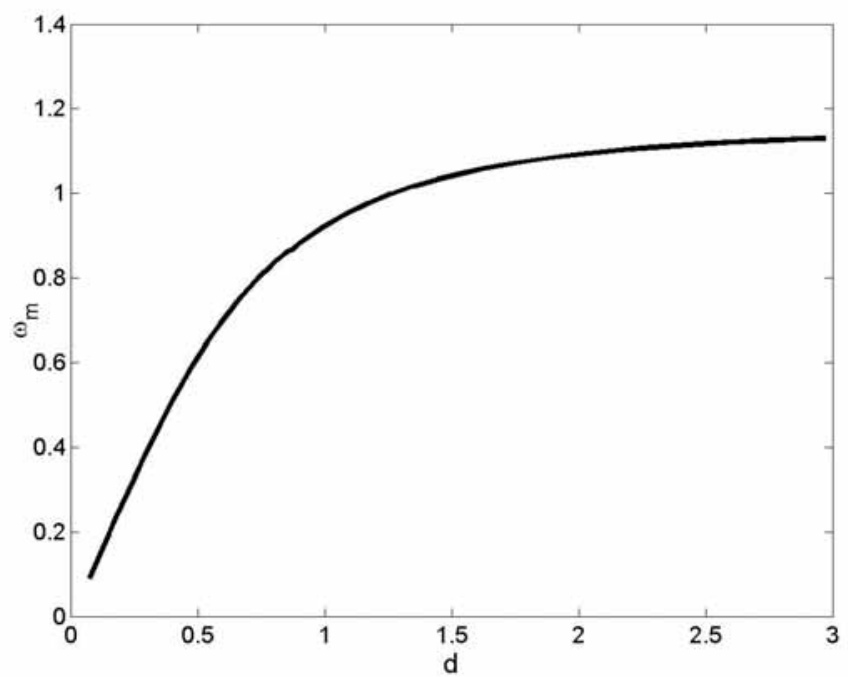

Fig. 24. Optimum curve in the $\omega_{m}$ - $d$ plane.

This follows suit with the line of minima curve illustrated in Fig. 20. From the analysis of these figures it becomes obvious that to maintain a minimum of acceleration transmission across the frequency spectrum that larger amplitude constraints must be accepted. However, if only very low frequency excitations are of concern it may be advantageous to employ a slightly higher damping to minimize displacement transmissibility while keeping in mind that this entails larger acceleration transmission at higher frequencies. More specifically, from Figs 28 through 30 it becomes obvious that there exists some critical value of the inertia track cross-sectional area noting that this term is the term that most readily affects the damping of the mount. If the area of the inertia track is less than the critical area value it becomes obvious that the mount will exhibit excessive damping and fail to provide the appropriate switching mechanism. However, if the area of the inertia track is too large, the mount may be under damped and not provide sufficient isolation during primary resonance. 


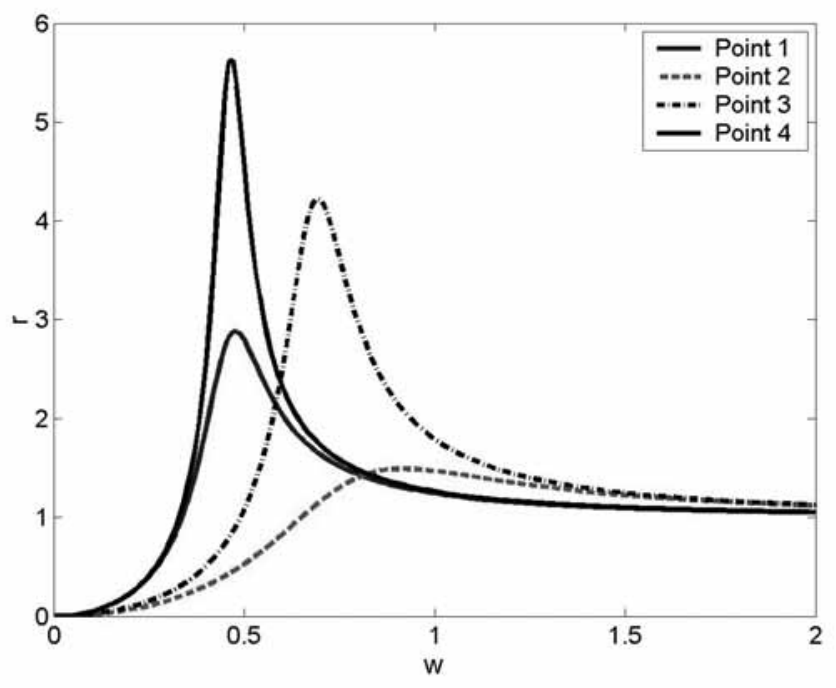

Fig. 25. Optimized relative displacement frequency responses.

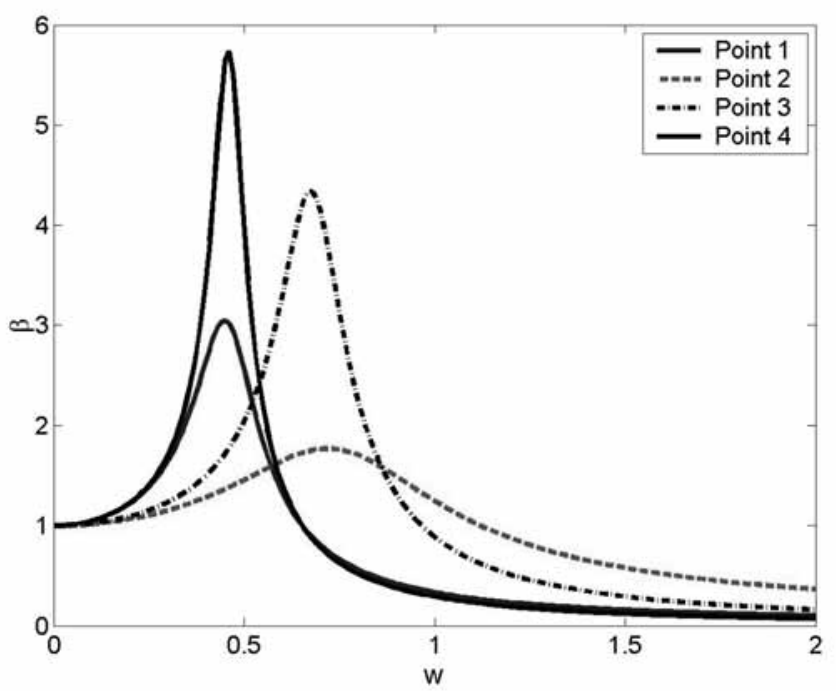

Fig. 26. Optimized absolute displacement frequency response.

\section{Transient response}

Many times in problems relating to vibrating systems knowledge of the transient response of the system is desirable to gain insight on system behavior due to random excitations. Therefore, it becomes desirable to see how optimization in the frequency domain affects the transient behavior of the system to a step input. To determine the transient responses of the hydraulic mount, the fourth order Runge-Kutta method was employed to solve the equations of motion numerically. The transient response was calculated by imposing a step input to the initial position of the engine mass.

Figures 31 and 32 illustrate the response of the engine mass due to a step input. Figure 31 compares the transient responses of the following points: point $1\left(d=0.201, \omega_{m}=0.26369\right)$, point $2\left(d=0.701, \omega_{m}=0.77793\right)$, point 


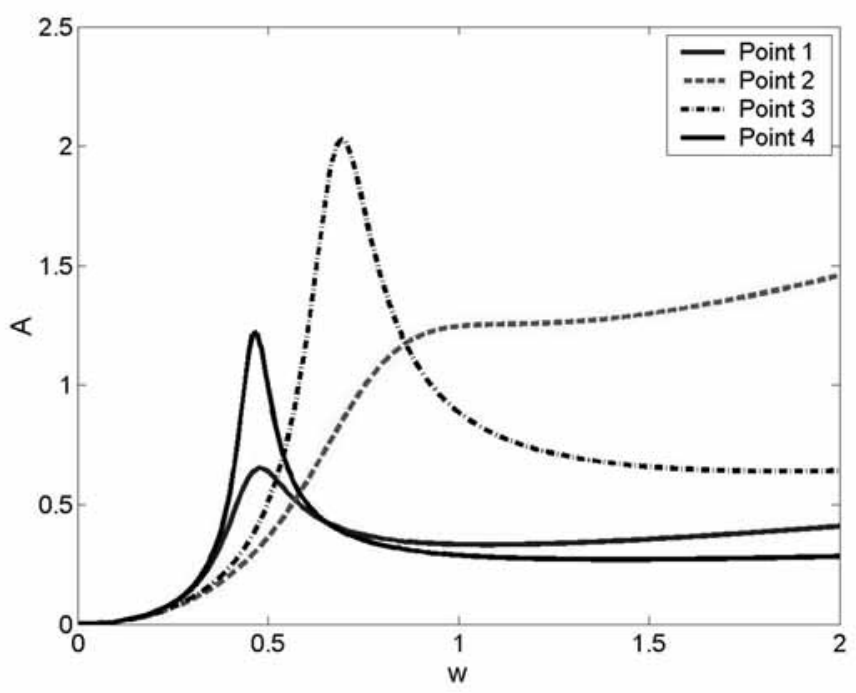

Fig. 27. Optimized absolute acceleration frequency response.

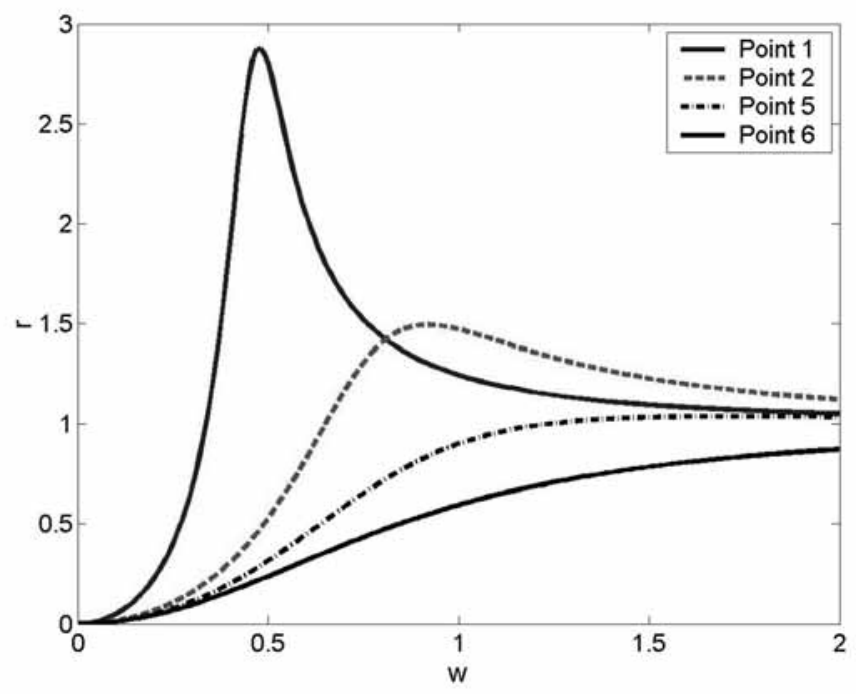

Fig. 28. Optimized relative displacement frequency response.

$3\left(d=0.201, \omega_{m}=0.5760\right)$, and point $4\left(d=0.101, \omega_{m}=0.26369\right)$ for engine mass displacement, where as Fig. 31 compares the transient acceleration response. As is readily apparent, the oscillation of the system defined by point 2 dies the fastest, but this system also exhibits the highest damping of the four points compared. The transient responses of the systems defined by points 1 and 4 illustrate the same natural frequency, but different damping. However, these points show almost identical transient responses. Both of these systems oscillations die relatively quickly, however they do not die as rapidly as the system defined by point 1 . The system defined by point 3 takes the longest to die, and in comparison to the frequency responses of this system, it appears to be less than ideal for consideration as a design concept.

Figures 33 and 34 illustrate the internal mount behavior of the same four points due to a step input. The internal behavior of the mount becomes important in verifying the functionality of the inertia track and decoupler components 


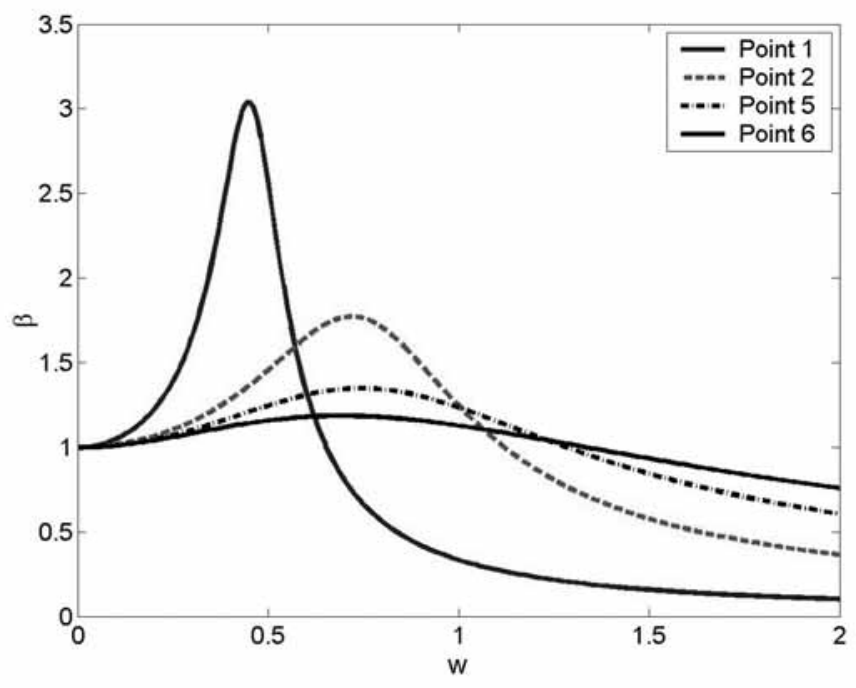

Fig. 29. Optimized absolute displacement frequency response.

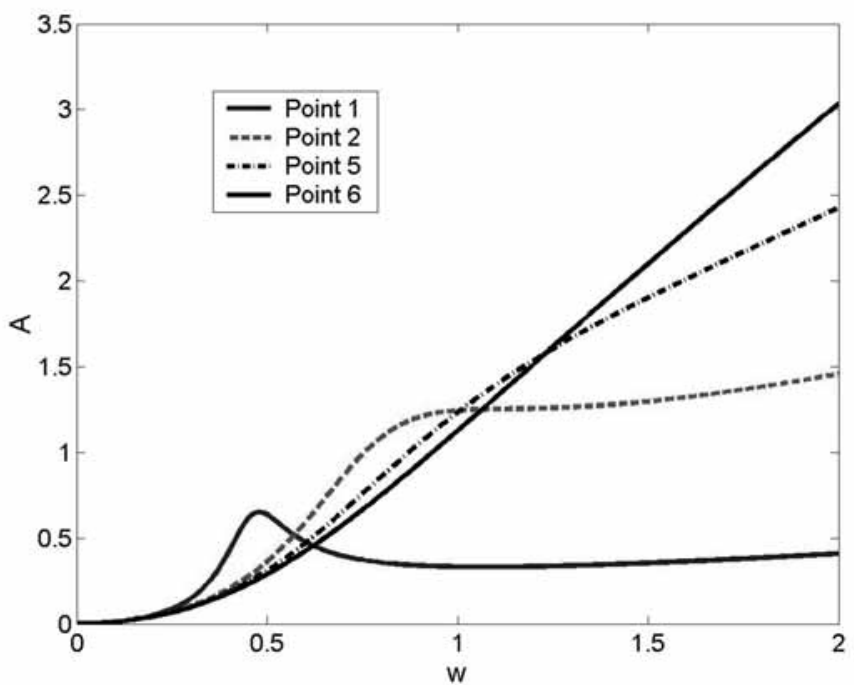

Fig. 30. Optimized absolute acceleration frequency response.

after the optimization analysis.

Figure 33 illustrates the flow rate through the decoupler for the four points under consideration. Here it may be seen that all points cause the closing of the decoupler, signified by a bounding of the flow rate through the decoupler. However, it becomes obvious that the system governed by point 2 never allows the decoupler to open; therefore, the only flow between chambers is through the inertia track.

Figure 34 illustrates the flow through the inertia track, and as before, the time domain solutions for points 1 and 4 coincide. In addition, the flow rate through the inertia track of the system defined by point 2 can be observed to have the smallest amplitude. This is due to the higher overall damping of the system because of the smaller cross-sectional area of the inertia track. Therefore, the flow rate through the inertia track is less than the other systems investigated.

Figure 35 illustrates the pressure difference between the fluid chambers of four different configurations of the 


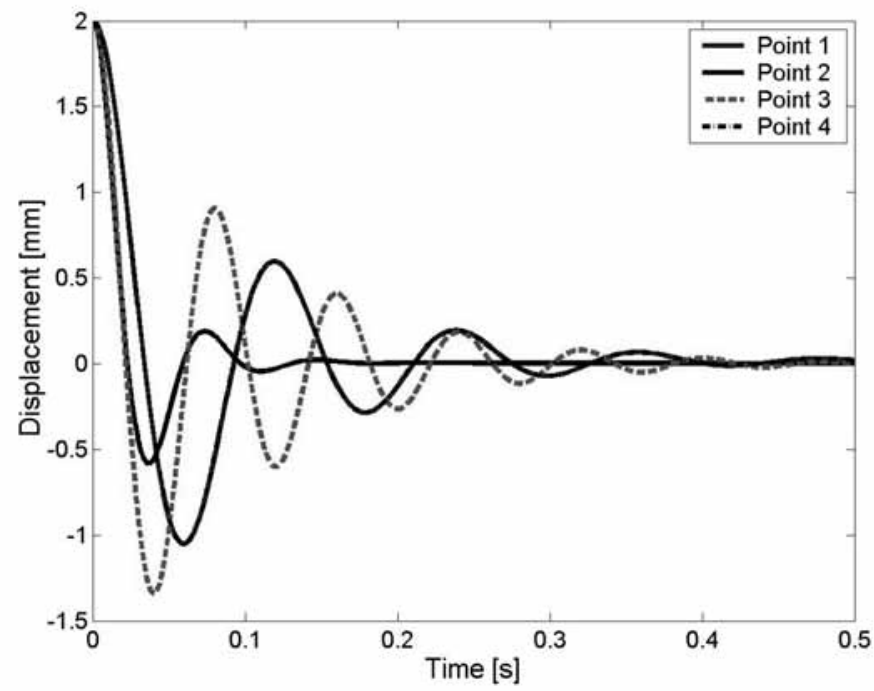

Fig. 31. Optimized engine mass displacement transient response.

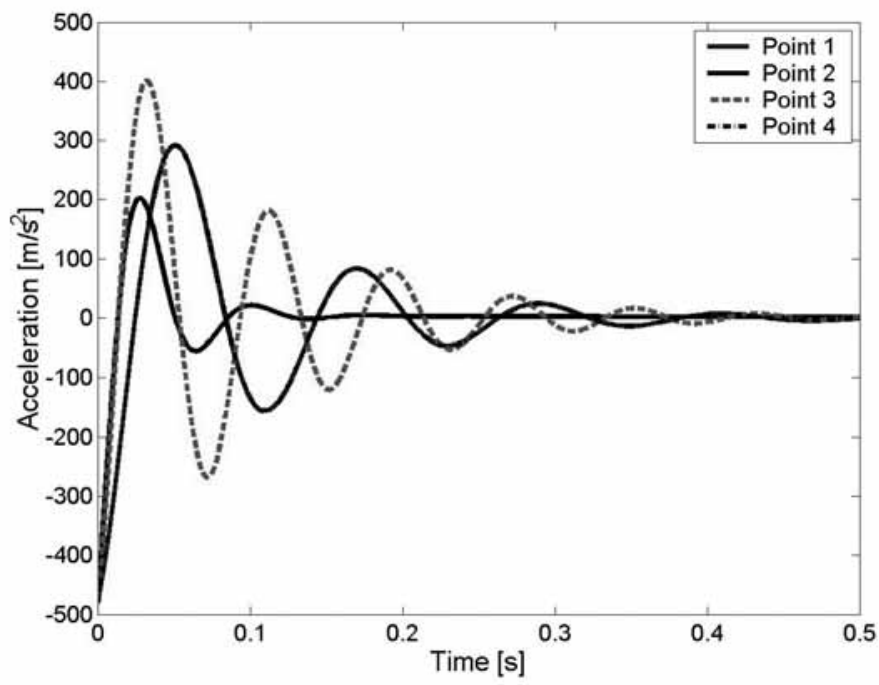

Fig. 32. Optimized engine mass acceleration transient response.

mount undergoing a step input. Notice that because the decoupler is almost always closed (see Fig. 33) the pressure difference between chambers must be equalized via the inertia track. Therefore, after comparison between Figs 34 and 35 it may be seen that the pressure difference slightly leads the inertia track flow noting it is the pressure difference that drives the flow through the fluid passages.

\section{Conclusion}

This paper has shown that an optimum hydraulic engine mount system can be determined in a frequency domain using an RMS averaging of the frequency response functions. The results of the RMS averaging provide insight 


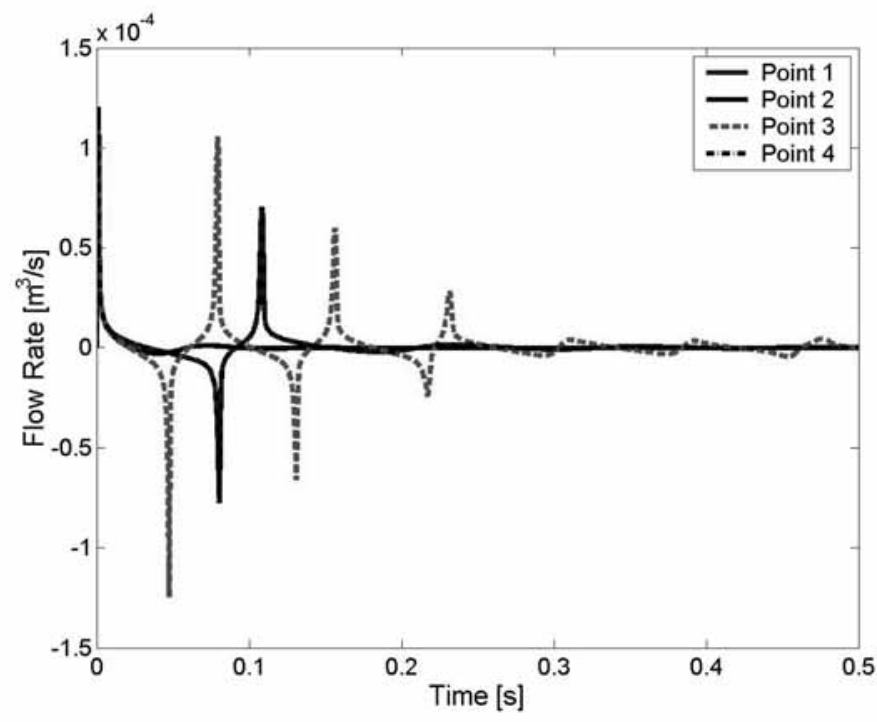

Fig. 33. Optimized decoupler flow rate transient response.

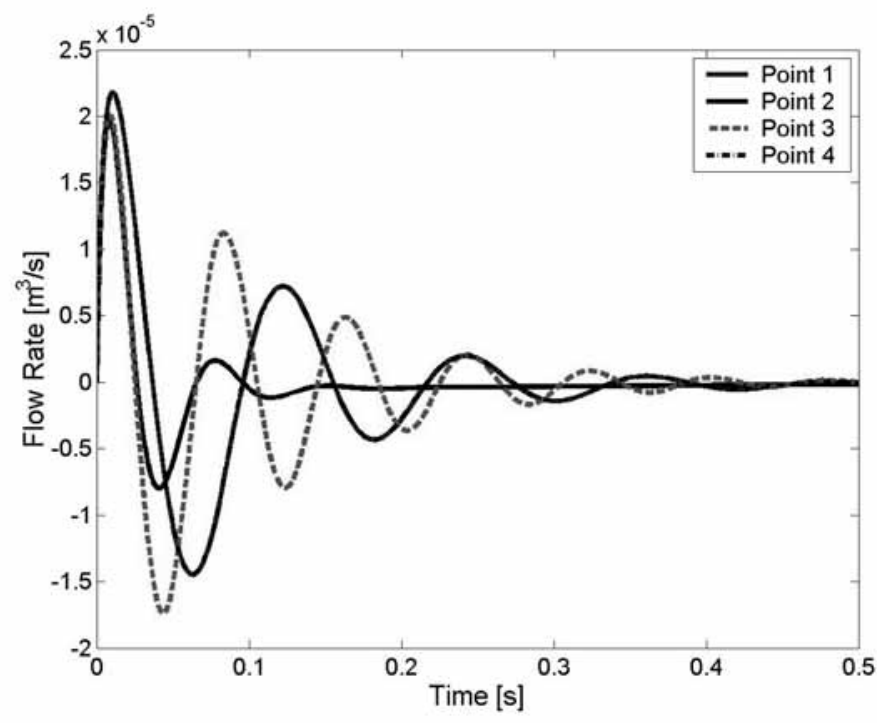

Fig. 34. Optimized inertia track flow rate transient response.

that allows the development of a design chart by which appropriate mount parameters may be selected based upon space/displacement constraints or acceleration constraints. It has also been shown that using the RMS averaging technique results in a line of minima illustrating an optimum relationship between acceleration and displacement transmissibility. This line of minima is defined by a series of points consisting of different mount parameters. It has also been shown that to influence the overall damping of the system, the inertia track becomes the critical component; where as the decoupler geometry has the greatest impact upon the primary resonant domain. More specifically, the decoupler geometry contributes to the location of the primary resonant domain and hence the location of the switching point between damping modes. In addition to exploring the mount behavior in the frequency domain, the transient response of the system also was investigated. This analysis showed that not only does the RMS averaging technique provide an optimized solution in the frequency domain, but also provides a system with an improved 


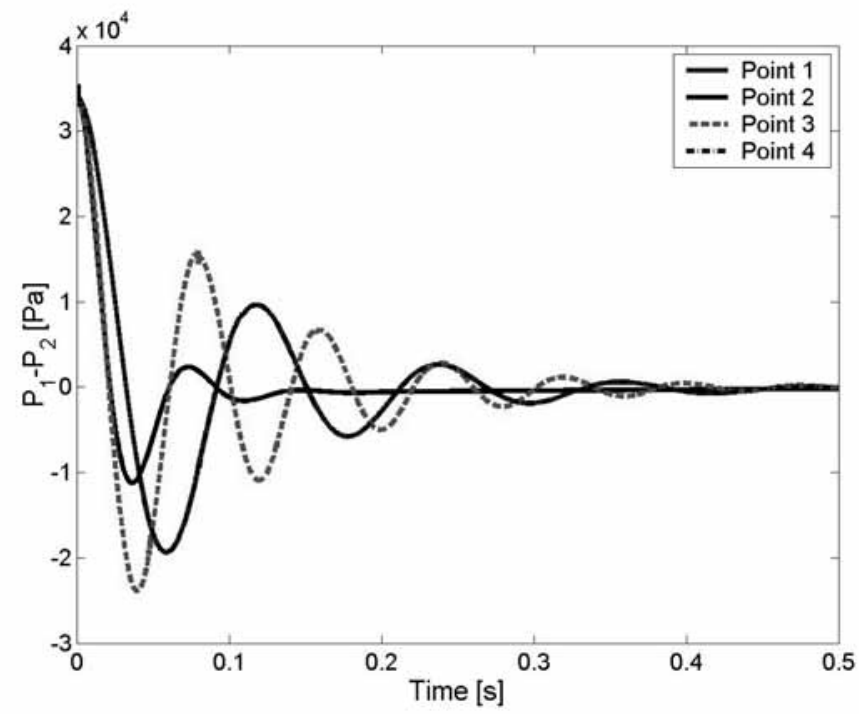

Fig. 35. Chamber pressure difference transient responses.

transient response.

\section{Nomenclature}

$\begin{array}{lll}A & = & \text { Area } \\ B & = & \text { Equivalent viscous damping coefficient } \\ C & = & \text { Volumetric compliance } \\ E & = & \text { Nonlinear coefficient } \\ H & = & \text { Step size } \\ N & = & \text { Flow resistance } \\ D & = & \text { Hydraulic diameter } \\ j & = & \text { Complex number } \\ K=1 / C_{1}+1 / C_{2} & = & \text { Inverse sum of compliances } \\ K_{r} & = & \text { Upper rubber equivalent stiffness } \\ L & = & \text { Length } \\ M & = & \text { Mass } \\ P & = & \text { Pressure } \\ Q & = & \text { Flow rate } \\ R & = & \text { RMS of acceleration transmissibility } \\ T & = & \text { Period } \\ t & = & \text { Time } \\ x & = & \text { Position } \\ Y & = & \text { Excitation amplitude } \\ z & = & \text { Relative position } \\ \Delta & = & \text { Gap size } \\ \eta & = & \text { RMS of relative displacement transmissibility } \\ \mu & = & \text { Dynamic viscosity } \\ \rho & = & \text { Fluid density } \\ \omega & = & \text { Dxcitation frequency } \\ \xi & & \\ & & \\ & = & \\ & = & \end{array}$

Nondimensional parameters: $\tau=\Omega t$

Nondimensional time

$\Omega^{2}=A_{d}^{2} K / M_{d} \quad=$

Nondimensional parameter 


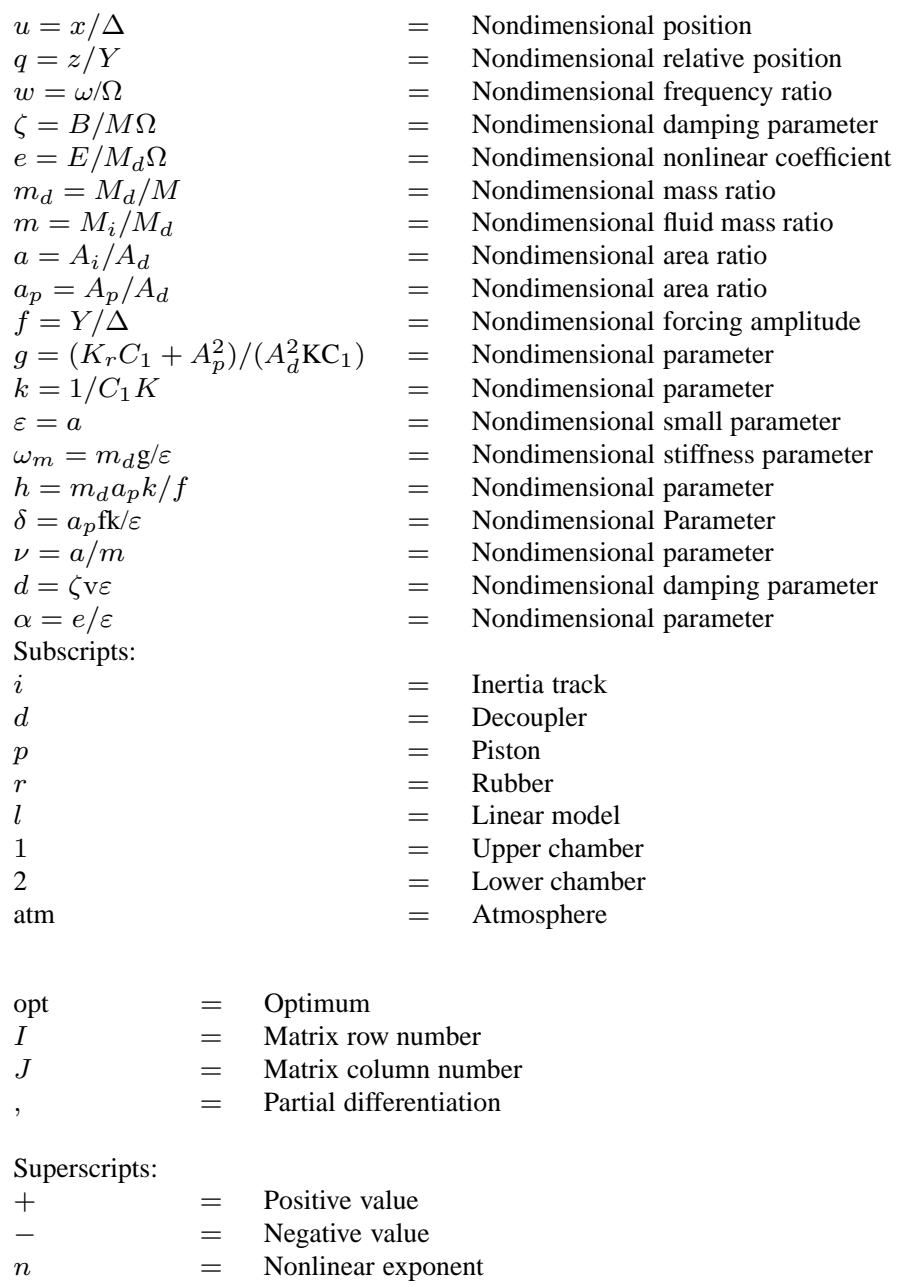

\section{Appendix A}

\section{Time derivatives:}

$$
\begin{aligned}
& \begin{array}{l}
d r(\tau) \\
d \tau
\end{array}={ }_{2 w}^{1}\left(Z_{1} r(\tau)-Z_{2} h r_{d}(\tau)-Z_{3} \varepsilon h r_{i}(\tau)\right) \\
& r(\tau) \stackrel{d \phi(\tau)}{d \tau}={ }_{2 w}^{1}\left(Z_{4} r(\tau)-Z_{5} h r_{d}(\tau)-Z_{6} \varepsilon h r_{i}(\tau)+Z_{7}\right)
\end{aligned}
$$

where:

$$
\begin{aligned}
& Z_{1}=\varepsilon\left(\omega_{m} \sin (2 \psi)+d w \cos (2 \psi)-d w\right)-w^{2} \sin (2 \psi) \\
& Z_{2}=\sin \left(\psi+\psi_{d}\right)-\sin \left(\psi-\psi_{d}\right) \\
& Z_{3}=\sin \left(\psi+\psi_{i}\right)+\sin \left(\psi-\psi_{i}\right)+w^{2}(\cos (\phi(\tau))-\cos (\phi(\tau)-2 \psi))
\end{aligned}
$$




$$
\begin{aligned}
& Z_{4}=\varepsilon\left(\omega_{m}(1+\cos (2 \psi))-d w \sin (2 \psi)\right)-w^{2}(1+\cos (2 \psi)) \\
& Z_{5}=\cos \left(\psi-\psi_{d}\right)+\cos \left(\psi+\psi_{d}\right) \\
& Z_{6}=\cos \left(\psi-\psi_{i}\right)+\cos \left(\psi+\psi_{i}\right) \\
& Z_{7}=w^{2}(\sin (\phi(\tau))+\sin (\phi(\tau)-2 \psi))
\end{aligned}
$$

\section{Phase angle equations:}

$$
\begin{aligned}
& \phi=\arctan \left(\begin{array}{c}
\varepsilon \omega_{m}-w^{2} \\
\varepsilon w d
\end{array}\right) \\
& \gamma=\arctan \left(\begin{array}{c}
\varepsilon \omega_{m}^{2}-\omega_{m} w^{2}+\varepsilon d^{2} w^{2} \\
d w^{3}
\end{array}\right)
\end{aligned}
$$

\begin{tabular}{|c|c|c|c|c|c|c|c|}
\hline \multicolumn{8}{|c|}{ Optimum values: } \\
\hline$R$ & $\eta$ & $\omega_{m}$ & $d$ & $R$ & $\eta$ & $\omega_{m}$ & $d$ \\
\hline 0.040498 & 1.4248 & 0.029253 & 0.026 & 1.0038 & 1.0208 & 0.82458 & 0.776 \\
\hline 0.082306 & 1.4314 & 0.060029 & 0.051 & 1.0161 & 1.0051 & 0.83869 & 0.801 \\
\hline 0.12835 & 1.4402 & 0.094361 & 0.076 & 1.0275 & 0.98959 & 0.85204 & 0.826 \\
\hline 0.17328 & 1.4352 & 0.12783 & 0.101 & 1.0382 & 0.97436 & 0.86481 & 0.851 \\
\hline 0.22014 & 1.4286 & 0.16231 & 0.126 & 1.048 & 0.95941 & 0.87689 & 0.876 \\
\hline 0.26549 & 1.4186 & 0.19611 & 0.151 & 1.0572 & 0.94473 & 0.88844 & 0.901 \\
\hline 0.3107 & 1.4073 & 0.2299 & 0.176 & 1.0657 & 0.93033 & 0.89937 & 0.926 \\
\hline 0.35575 & 1.3953 & 0.26369 & 0.201 & 1.0736 & 0.9162 & 0.90982 & 0.951 \\
\hline 0.3994 & 1.382 & 0.29683 & 0.226 & 1.0775 & 0.90154 & 0.915 & 0.976 \\
\hline 0.44228 & 1.3682 & 0.32962 & 0.251 & 1.0862 & 0.88826 & 0.92666 & 1.001 \\
\hline 0.48426 & 1.3537 & 0.36197 & 0.276 & 1.1095 & 0.83693 & 0.9607 & 1.101 \\
\hline 0.52465 & 1.3387 & 0.39354 & 0.301 & 1.1266 & 0.78992 & 0.98847 & 1.201 \\
\hline 0.56349 & 1.3231 & 0.42431 & 0.326 & 1.1396 & 0.74688 & 1.0116 & 1.301 \\
\hline 0.6011 & 1.3071 & 0.45442 & 0.351 & 1.1496 & 0.70748 & 1.0311 & 1.401 \\
\hline 0.63717 & 1.2908 & 0.4837 & 0.376 & 1.1571 & 0.67148 & 1.0473 & 1.501 \\
\hline 0.67154 & 1.2742 & 0.51205 & 0.401 & 1.1629 & 0.63854 & 1.061 & 1.601 \\
\hline 0.70423 & 1.2574 & 0.53946 & 0.426 & 1.1674 & 0.60836 & 1.0726 & 1.701 \\
\hline 0.73526 & 1.2403 & 0.56591 & 0.451 & 1.1709 & 0.58063 & 1.0826 & 1.801 \\
\hline 0.76463 & 1.2232 & 0.59139 & 0.476 & 1.1737 & 0.55512 & 1.0912 & 1.901 \\
\hline 0.79238 & 1.2059 & 0.6159 & 0.501 & 1.176 & 0.53159 & 1.0986 & 2.001 \\
\hline 0.81853 & 1.1886 & 0.63944 & 0.526 & 1.1778 & 0.50982 & 1.1051 & 2.101 \\
\hline 0.84312 & 1.1713 & 0.662 & 0.551 & 1.1792 & 0.48964 & 1.1108 & 2.201 \\
\hline 0.86617 & 1.1541 & 0.68359 & 0.576 & 1.1804 & 0.47092 & 1.1158 & 2.301 \\
\hline 0.88775 & 1.1369 & 0.70423 & 0.601 & 1.1814 & 0.4535 & 1.1202 & 2.401 \\
\hline 0.90796 & 1.1198 & 0.72397 & 0.626 & 1.1823 & 0.43726 & 1.1242 & 2.501 \\
\hline 0.92693 & 1.1028 & 0.74285 & 0.651 & 1.1829 & 0.42209 & 1.1277 & 2.601 \\
\hline 0.94457 & 1.086 & 0.76083 & 0.676 & 1.1835 & 0.4079 & 1.1308 & 2.701 \\
\hline 0.96095 & 1.0694 & 0.77793 & 0.701 & 1.184 & 0.3946 & 1.1336 & 2.801 \\
\hline 0.97631 & 1.053 & 0.79427 & 0.726 & 1.1842 & 0.3821 & 1.135 & 2.901 \\
\hline 0.99056 & 10368 & 080982 & 0751 & & & & \\
\hline
\end{tabular}

\section{Appendix B}

\section{References}

[1] J.E. Colgate, C.T. Chang, Y.C. Chiou, W.K. Kiu and L.M. Keer, Modeling of a hydraulic engine mount focusing on response to sinusoidal and composite excitations, Journal of Sound and Vibration184 (1995), 503-528. 
[2] A. Geisberger, A. Khajepour and F. Golnaraghi, Non-linear modeling of hydraulic mounts: Theory and experiment, Journal of Sound and Vibration 249 (2002), 371-397.

[3] G. Nakhaie Jazar and Golnaraghi, M. Farid, Nonlinear modeling, experimental verification, and theoretical analysis of a hydraulic engine mount, Journal of Vibration and Control 8(1) (2002), 87-116.

[4] J.P. Den Hartog, Mechanical vibrations, (4th ed.), McGraw Hill, New York, 1956.

[5] G. Nakhaie Jazar and Golnaraghi M. Farid, Engine mounts for automotive application: A survey, The Shock and Vibration Digest 34, (2002), 363-379.

[6] F. Golnaraghi and G. Nakhaie Jazar, Development and analysis of a simplified nonlinear model of a hydraulic engine mount, Journal of Vibration and Control 7 (2001), 495-426.

[7] A.H. Nayfeh and D. Mook, Nonlinear oscillations, John Wiley, New York, 1979.

[8] R.J. Schilling and S.L. Harris, Applied numerical methods for engineers, Brooks/Cole, Pacific Grove, Ca, 2000.

[9] Hanselman, Duane, Littlefield, Bruce, Mastering MATLAB 6 A comprehensive tutorial and reference, Prentice Hall, Upper Saddle River, NJ, 2001.

[10] G. Nakhaie Jazar, A. Narimani, M.F. Golnaraghi and D.A. Swanson, Practical frequency and time optimal design of passive linear vibration isolation mounts, Journal of Vehicle System Dynamics 39(6) (2003), 437-466.

[11] G. Nakhaie Jazar, Stability chart of parametric vibrating systems using energy-rate method, International Journal of Nonlinear Mechanics 39(8) (2004), 1319-1331,

[12] G. Kim and R. Singh, A study of passive and adaptive hydraulic engine mount systems with emphasis on non-linear characteristics, Journal of Sound and Vibration 179 (1995), 427-453.

[13] H. Adiguna, M. Tiwari, R. Singh, H.E. Tseng and D. Hrovat, Transient response of a hydraulic engine mount, Journal of Sound and Vibration 268 (2003), 217-248. 

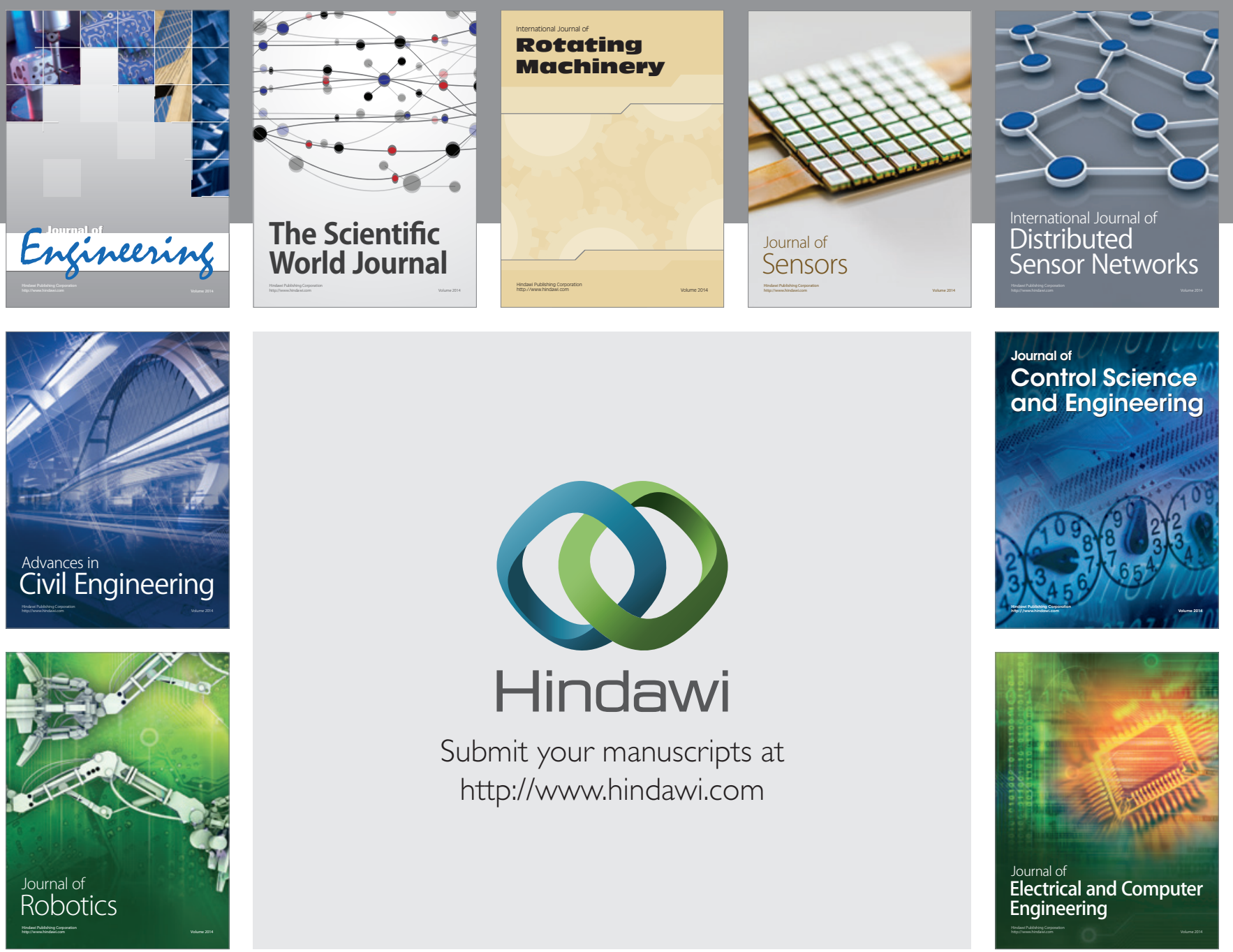

Submit your manuscripts at

http://www.hindawi.com
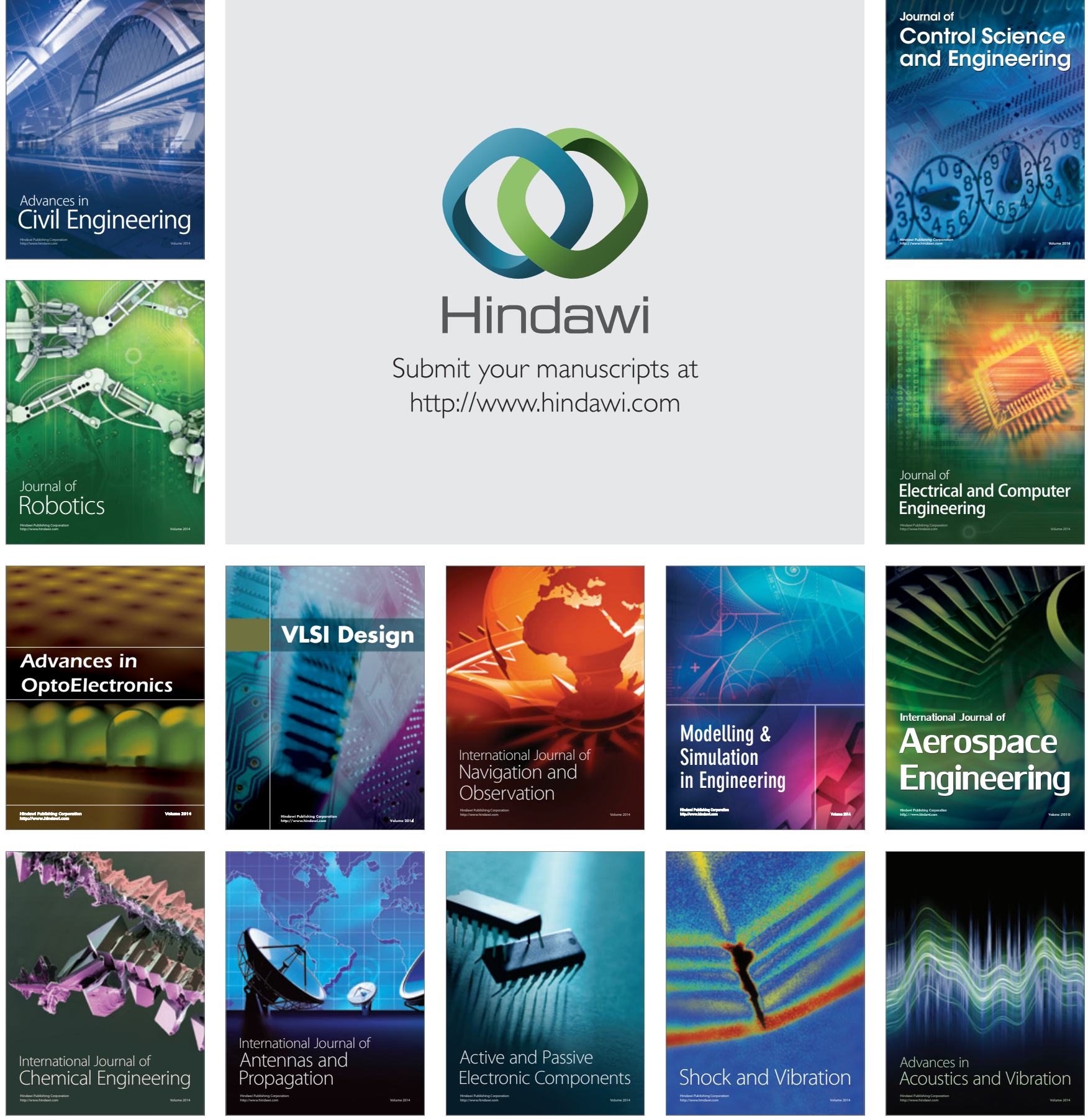